\title{
Metropole - Größe, Funktion und Symbolik. Eine quantitative Textanalyse deutscher Printmedien für Berlin und Hamburg
}

\section{Metropolis - size, function and symbolism. A quantitative text analysis of German print media for Berlin and Hamburg}

https://doi.org/10.2478/rara-2019-0060

Eingegangen: 12. März 2019; Angenommen: 16. Oktober 2019

Kurzfassung: Als Inbegriff des Urbanen erlebt der Stadttypus „Metropole“ seit nunmehr zwei bis drei Jahrzehnten eine fortwährende Konjunktur. Mit einer quantitativen Textanalyse der führenden deutschen überregionalen Printmedien werden die Muster des Sprachgebrauchs für den Begriff Metropole und für die beiden größten Städte Berlin und Hamburg herausgearbeitet. Sie werden als Bedeutungszuschreibungen in massenmedialen Kommunikationsprozessen interpretiert und erzeugen ein semantisches Feld, dessen Analyse Aussagen über Größe, Funktion und Symbolik einer Metropole auch im Zeitverlauf zulässt.

Schlüsselwörter: Metropole, Lexikometrie, Printmedienanalyse, Hamburg, Berlin

Abstract: As the epitome of the urban, the city type "metropolis" has been experiencing an ongoing boom for two to three decades. With a quantitative text analysis of the leading German national print media, the patterns of linguistic use for the term metropolis and for the two largest cities Berlin and Hamburg are highlighted. They are interpreted as attributions of meaning in mass media communication processes and generate a semantic field whose analysis allows declarations to be made about size, function and imagery of a metropolis over time.

Keywords: Metropolis, Lexicometry, Print media analysis, Hamburg, Berlin

*Corresponding author: Dr. Kati Volgmann, ILS - Institut für Landes- und Stadtentwicklungsforschung, Brüderweg 22-24, 44135 Dortmund, Deutschland, E-Mail: kati.volgmann@ils-forschung.de, ORCID: 0000-0002-5095-3374 


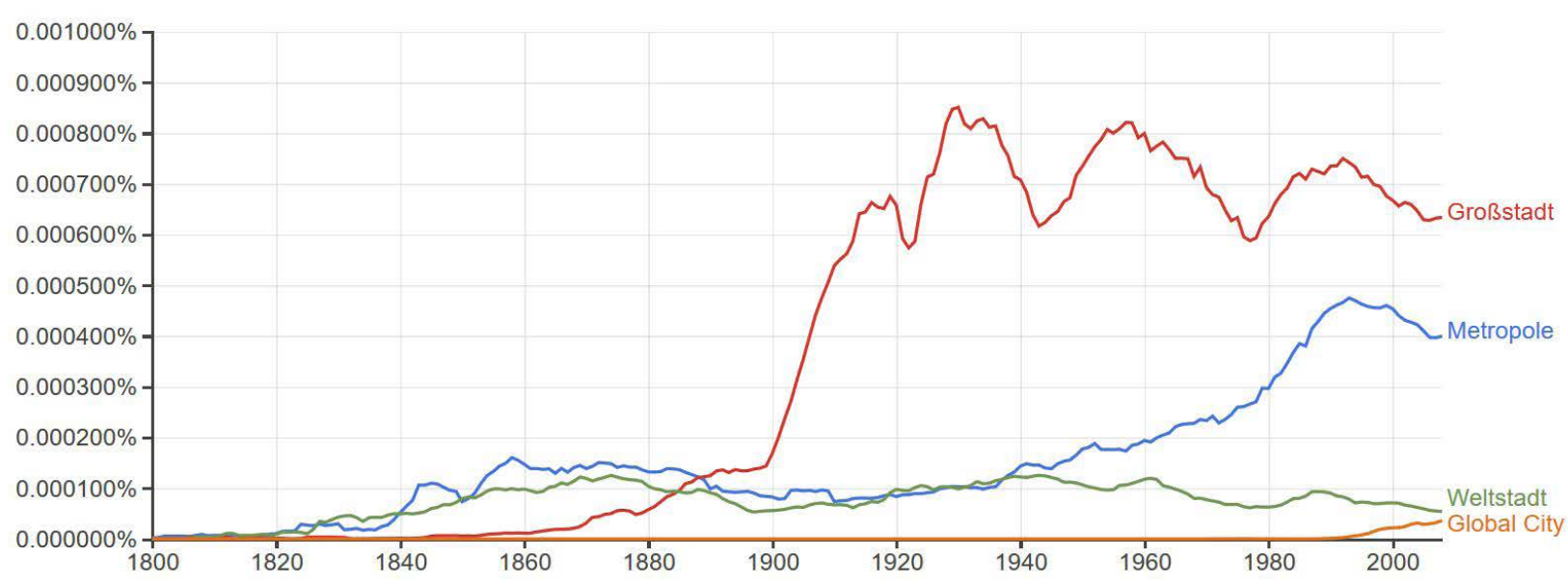

Abbildung 1: Nennungen von Großstadtbegriffen im Google Books Ngram Viewer 1800-2008² Quelle: https://books.google.com/ngrams

\section{Konjunktur des Metropolenbegriffs}

Als Inbegriff des Urbanen hat der Begriff „Metropole“ seit den 1990er-Jahren sowohl in den Medien, der Werbung, im Standortmarketing, in der Wissenschaft als auch in der Raumordnungspolitik eine erstaunliche Konjunktur. Das bestätigt auch ein Blick in den Google Books Ngram Viewer ${ }^{1}$ über den Begriff Metropole und weitere Großstadtbegriffe (vgl. Abbildung 1).

Als Chiffre für Modernität, Kreativität, Weltläufigkeit und Vielfalt verheißt der Stadttypus Metropole ein Bild der urbanen Faszination, welches unsere Vorstellung von Urbanität diskursiv rahmt. Metropole hebt sich daher durch ein „Mehr" (Hose/Levin 2009: 9) an Bevölkerung, Ressourcen, Aufmerksamkeit, Kultur gegenüber Mittelmaß und Provinzialismus ab. An Metropolen haften Vorstellungen, Wünsche, Weltstadtbilder, die sich unter anderem in der Semantik der Titulierung als "Laboratorien der Moderne" (Matejovski 2000) ausdrücken.

Metropolen dienen als Schauplätze in Film und Fernsehen, häufig als Kulisse oder Mittelpunkt der Handlung, oder dienen als Requisite in Werbekampagnen globa-

1 Der "Google Ngram Viewer" ist im Rahmen des Projektes "Google Books" entstanden und analysiert mittels Data Mining Nennungen von Wortfolgen in gedruckten Publikationen. Für die vorliegende Untersuchung wurden die deutschsprachigen gedruckten Publikationen seit 1800 quantifiziert.

2 Die y-Achse zeigt an, wieviel Prozent des deutschen Korpus aus dem gewählten n-gram bestehen. Gezählt wird dabei nicht die Anzahl der einzelnen Zeichen im Korpus, sondern die Anzahl von Wortformen. ler Auto-, Mode- und Kosmetikmarken, die exklusiven weltstädtischen und metropolitanen Wert versprechen. Feuilletons überregionaler Zeitungen, populärwissenschaftliche Magazine küren regelmäßig die kreativsten, lebenswertesten, teuersten, optimistischsten oder dreckigsten Metropolen der Welt (z. B. Nitt-Drießelmann/ Wedemeier 2017). ${ }^{3}$

Der intensiv geführte gesellschaftliche und wissenschaftliche Diskurs in der nationalen (Blotevogel 2000; Danielzyk 2012; Mieg 2012a) und internationalen Stadtforschung (u. a. Sassen 1991; Taylor/Walker/Beaverstock 2002) hat sich in den 1990er-Jahren auf die deutsche Raumordnungspolitik übertragen. Mit der Ausrufung der „Europäischen Metropolregionen“ 1995 durch die Ministerkonferenz für Raumordnung hat der Begriff kontinuierlich an Bedeutung gewonnen (BMRBS 1995: 27). Insbesondere die höchste Stufe städtischer Zentralität - Metropole zu sein, das Etikett Metropole zu verwenden oder zumindest metropolitane Eigenschaften zu besitzen - scheint für viele Städte erstrebenswert.

Was aber ist eine Metropole? Ist es ein Zentrum eines Weltreichs, die höchste Zentralität im Städtesystem, eine Hauptstadt, eine Weltstadt oder doch nur ein Mythos, der sich gar nicht fassen lässt? Setzt man sich mit den Diskursen zu Metropolen auseinander, fällt auf, dass das Wort im Laufe der Zeit einen Bedeutungswandel vollzogen hat und die wissenschaftlichen Bemühungen um die Klärung und theoretische Einordnung des

3 Vgl. auch den „Time Out Index“ 2019; https://www.timeout.com/ london/citylifeindex (05.09.2019) sowie das Städteranking von Wirtschaftswoche, Immobilienscout und IW Consult 2017; https:// www.wiwo.de/themen/staedte-ranking (05.09.2019). 
Metropolenbegriffs in der Stadtforschung stark auseinanderdriften und nicht zu einer einheitlichen Metropolentheorie geführt haben. Schon die Frage, welche Größe eine Metropole im Vergleich zu einer Mega-City einnimmt oder ob ökonomische Funktionen wichtiger sind als kulturelle Funktionen, kann nicht eindeutig beantwortet werden. Gründe dafür liegen möglicherweise in der semantischen Vieldeutigkeit des Begriffs.

Der Beitrag setzt sich zunächst mit einer Systematisierung verschiedener Dimensionen des Metropolenbegriffs auseinander (Kapitel 2), die das konzeptionelle Gerüst für die empirische Analyse bilden. Die Lexikometrie - eine Akzentuierung der empirisch ausgerichteten Diskursanalyse - wird als quantitatives Verfahren eingesetzt (Kapitel 3), die innerhalb der deutschen Printmedien semantische Zuschreibungen, Raumbilder, Eigenschaften herausfiltert und neue Informationen darüber generiert, wie der Begriff in der Alltagssprache verwendet wird, ob die Zuschreibungen vom wissenschaftlichen Diskurs abweichen und welche Städte zu Metropolen erhoben werden (Kapitel 4).

Die Ergebnisse werden in Bezug zu Analysen über die beiden ,deutschen Metropolen' Berlin und Hamburg gestellt und die Unterschiede zwischen metropolitanen, aber auch stadt- bzw. lokalspezifischen Zuschreibungen sowie die Verschiebungen vor und nach 1990 herausgearbeitet (Kapitel 4.3). Abschließend werden die Ergebnisse um die begriffliche Einordnung im Zusammenhang mit deren Bedeutung für die planerische Praxis diskutiert (Kapitel 5).

\section{Zum Metropolenbegriff}

Innerhalb der Stadtforschung existieren verschiedene theoretische und konzeptionelle Zugänge, um den Metropolenbegriff näher zu fassen. An dieser Stelle werden die unterschiedlichen Zugänge und die semantischen Verschiebungen im Zeitverlauf beleuchtet. Anschließend werden drei Dimensionen als konzeptionelles Gerüst für die Systematisierung der empirischen Printmedienanalyse abgeleitet.

\subsection{Entstehung und Entwicklung des Metropolenbegriffs bis zur Industrialisierung}

Ursprünglich leitet sich Metropole aus dem altgriechischen Wort metropolis (= Mutterstadt) ab, von der aus andere Städte als Kolonien gegründet wurden (Kemper
2006: 2; Mieg 2012a: 13). Historische Zeugnisse der griechischen Literatur geben an, dass die metropolis wichtige religiöse, kulturelle und politische Funktionen übernahm (Hose/Levin 2009: 13 f.). War in der Antike die griechische Hafenstadt Syrakus eine Metropole, war das Imperium Romanum mit Rom als Hauptstadt in der Spätantike das politische, militärische und kirchliche Machtzentrum. Mit dem Ende der römischen Antike und dem Bedeutungsgewinn der weströmischen Kirche wandelte sich Roms Semantik und wurde nun bis weit in die Neuzeit mit kirchlichen und religiösen Attributen gleichgesetzt (Zohlen 1995; Mieg 2012b).

Der Begriff löste sich unter dem Einfluss der Urbanisierung und Industrialisierung im 19. Jahrhundert von der kirchengeschichtlich geprägten Konnotation zu einer stärkeren multifunktionalen Bedeutung (Engel/Lambrecht 1995: 26). Die enormen Wanderungsströme in die Städte veränderten nachhaltig die städtischen Strukturen und führten zu einer bislang nicht gekannten Größendimension der Städte. Zwischen dem 18. und 19. Jahrhundert entwickelte sich London mit 6,5 Millionen Einwohnern zur größten Industriestadt der Welt und erlebte damit einen Aufstieg zur ersten modernen Metropole, auch als Weltfinanzzentrum mit wichtigen Informations- und Managementfunktionen (Heineberg 2014: 376). Gegen Ende des 19. Jahrhunderts entwickelten sich vor allem in den USA neue Weltwirtschaftszentren. Damit begann der Aufstieg New Yorks, der Metropole des 20. Jahrhunderts.

Die unkontrolliert wuchernde enge Stadt der Industrialisierung griff Simmel (1903) mit dem Essay „Die Großstädte und das Geistesleben" auf und beschrieb Großstädte als moderne Orte des sozialen Lebens mit einer überaus großen Interaktionsdichte. Wettbewerb, Leistung, Konkurrenz bewirkten einen Schutzmechanismus gegenüber den maßlosen Sinneseindrücken und formten den „Typus des Großstädters“ (Helbrecht 2013: 169 f.). Großstädte galten in dieser Zeit als "Motor des Fortschritts", die nicht nur institutionelle Innovationen, sondern auch technische oder kulturelle Innovationen sei es die moderne Finanzwirtschaft, Börse, Tageszeitung, Architekten - hervorbrachten (Mieg 2012a: 17). Die spezifische Siedlungsform, städtische Ökonomie, kulturelle Innovationsfähigkeit und eine urbane Lebensweise machten nach Simmel (1903) das Wesen der Stadt aus.

Sein Werk wurde zum "theoretischen Steinbruch“ (Häußermann 2011: 17) der amerikanischen Stadtsoziologie, häufig allerdings in Form des Aufsatzes von Wirth (1938) „Urbanism as a Way of Life“ zitiert. Die Urbanitätsmerkmale Größe einer Stadt, Dichte des Siedlungsgebiets einer Stadt und Heterogenität der Bevölkerung 
fanden nach Wirth (1938) ihren stärksten Ausdruck in Metropolen. Urbanität als spezifischer way of life ist nicht nur an die Großstadt gebunden, dennoch, so Wirth (1938), sind es die Städte, die als Innovationszentren urbaner Lebensstile entstehen und von denen Urbanität ausgeht (vgl. Helbrecht 2013: 171). Zu dieser Zeit wurde auch weniger von Metropole, sondern eher von Weltstadt gesprochen, wenn ökonomische, kulturelle oder politische Funktionen gemeint waren. Ab den 1930erJahren bis etwa in die 1970er-Jahre hinein verschwand der Begriff Metropole in Deutschland fast völlig aus dem Sprachgebrauch (Zohlen 1995: 30).

\subsection{Rückkehr des Metropolenbegriffs als Ausdruck globaler Steuerungszentralen}

Globalisierung, Liberalisierung und Internationalisierung führen seit den letzten 30 Jahren zu einer neuen Form städtischer Zentralität, die die europäischen und nordamerikanischen Metropolen mit wichtigen Finanz-, Steuerungs- und Dienstleistungsfunktionen an die Spitze des globalen Städtesystems setzen (Sassen 1991; Blotevogel 2002; Heineberg 2014). Im Zusammenhang mit dem Globalisierungsprozess der Städte werden verschiedene Begriffe und Konzepte zu Weltstädten, World Cities, Globalizing Cities, Global Cities oder Global CityRegions (Gerhard 2004: 5) diskutiert, die in enger Verbindung zum Begriff Metropole stehen, aber nicht synonym verwendet werden dürfen. Kemper (2006: 3, 17) spricht sogar von einer „Renaissance des Metropolenbegriffs“.

Zwischen den Sprachräumen wird bei der Verwendung des Metropolenbegriffs unterschieden. In der englischsprachigen Stadtforschung findet man den wortwörtlich übersetzten Begriff metropolis so gut wie nie, stattdessen wird eher von big city oder giant city gesprochen (Lees 1984). Im Französischen wird statt von métropole oft nur von grande ville gesprochen (Blotevogel 2000: 145 f.). Die US-amerikanischen metropolitan areas, die englischen metropolitan counties oder die französischen métropoles d'équilibre oder régions métropolitaines entsprechen dagegen eher funktionalen Stadtregionen (Wiechmann 2009: 104). In der deutschen Stadtforschung steht der Metropolenbegriff dagegen stärker mit dem Weltstadtbegriff in Verbindung, obwohl der Begriff Metropole ebenfalls im Zusammenhang mit "großer Stadt" gebraucht wird (Ritter 1998: 51).

In unserer Alltagssprache wird er geradezu inflationär verwendet, wie beispielsweise die "Modemetropole" Düsseldorf, die „Bankenmetropole“ Frankfurt, die „Eurometropolen" Wien und Prag, die "Handelsmetropole“
Danzig oder die „Kunstmetropole“ Prag. Dies geschieht meist ohne eine Begriffsklärung und offensichtlich werden Städte als Metropolen bezeichnet, wenn einzelne zentrale Funktionen überregionale Bedeutung qualitativer Art bekommen (wie "Handelsmetropole") oder zu einer Region in Beziehung gesetzt werden (Engel/Lambrecht 1995: 25).

Die Auffassungen über Definitionen und Funktionen von Städten der höchsten Hierarchiestufe sind, wie der Begriff Metropole, sehr unterschiedlich. Die Ansätze vereint jedoch, dass sie sich auf die funktionale Charakteristik der Städte stützen, und gehen zurück auf Hall (1966), der die globale Reichweite und den weltstädtischen Charakter von World Cities als politische und ökonomische Zentren ihres Einflusses in den Mittelpunkt seines Ansatzes stellt. Hall führt die Entwicklung von World Cities auf die räumliche Trennung von Produktion und Management sowie neue Wirtschaftsbereiche und Produktionsprozesse sogenannter whitecollar jobs in unternehmensbezogenen Dienstleistungen zurück (Hall 1966: 27). In den 1980er-Jahren entwickelte Friedmann (1986) sieben Weltstadthypothesen, die die Bedeutung einer Stadt hinsichtlich ihrer Quantität und der Qualität funktionaler Eigenschaften einstufen. Weltstädte müssen demnach über internationale Unternehmenszentralen verfügen, die international in funktionaler Arbeitsteilung stehen. Dagegen basiert der Global-CityAnsatz der Stadtsoziologin Sassen (1991) auf einem systematischen Umstrukturierungsprozess der Wirtschaft hin zu einer Dienstleistungs- und Finanzwirtschaft. Der Global-City-Ansatz betont die enormen Wachstumspotenziale des global orientierten Finanz- und Dienstleistungskomplexes, der sich von der regionalen Produktionsbasis räumlich immer mehr abkoppelt. Die neue geänderte Zusammensetzung der Weltwirtschaft führte demnach zu einer Verlagerung des Dienstleistungs- und Finanzgewerbes in einzelne Städte, in denen Produktions- und Handelsschritte überwacht werden. Städte werden somit nicht isoliert betrachtet, sondern als strategische Standorte weltweiter Wirtschaftsoperationen interpretiert. Diese Sichtweise auf das urbane System als World City Network wurde von der Forschungsgruppe „Globalization and World Cities Study Group and Network" (GaWC) um Taylor, Walker und Beaverstock (2002), Beaverstock, Smith und Taylor (1999) sowie Hoyler (2004) weiterentwickelt und empirisch in einem interlocking network model umgesetzt, in dem sich die regionalen, nationalen und transnationalen Standortnetze global agierender wissensbasierter Dienstleistungen kreuzen (Taylor/Ni/Derudder et al. 2010). Daher liegen die Schwerpunkte der internationalen Stadtkon- 
zepte auch hauptsächlich auf den wirtschaftlichen Entscheidungs- und Kontrollfunktionen sowie Gatewayfunktion), die insbesondere bei den Global-City-Funktionen eine dominante Rolle einnehmen.

Für Deutschland ergibt sich das Problem, dass es keine Global City ersten Ranges hat. Frankfurt am Main ist aufgrund strategischer Finanz- und Gatewayfunktionen die bedeutendste Global City, wenn auch nur auf niedrigem Niveau (Derudder/Taylor 2016). Vor diesem Hintergrund wurde 1995 das Konzept der „Europäischen Metropolregion" in Deutschland von der Ministerkonferenz für Raumordnung beschlossen und für diese Räume in Anlehnung an funktional-qualitative Merkmale von Metropolen vier Metropolfunktionen entwickelt. Ausgangspunkt war der Beitrag von Bonneville (1994), der von der Annahme ausgeht, dass mehr Städte als nur die so genannten World Cities New York, Tokyo, London oder Paris internationalisierte Städte sein können (Bonneville (1994: 271, 278 ff.). Blotevogel (2002: 346) entwickelte daraus zunächst drei Metropolfunktionen (Entscheidungs- und Kontrollfunktion, Innovations- und Wettbewerbsfunktion und Gatewayfunktion) und später mit der Symbolfunktion die vierte Metropolfunktion mit verschiedenen Attributindikatoren. Diese Systematisierung hat Eingang in verschiedene Messkonzepte (BBSR 2011; Volgmann 2014) gefunden. Es zeigt sich, dass verschiedene Städte zu unterschiedlichen Zeitpunkten mal mehr oder mal weniger als Metropole wahrgenommen werden und somit gesellschaftlichen Veränderungen unterliegen. Im Laufe der Zeit verschiebt sich die Konnotation einer gesellschaftlichen Weltdeutung zu einer ökonomisierten Gesellschaft: Globale Macht und Ansehen werden nicht mehr durch Religion und Ideologien geprägt und weniger durch politische Macht, sondern eher durch Wohlstand, wirtschaftlich-technische Leistungsfähigkeit und technischen und wissenschaftlichen Fortschritt. Bis heute hält die funktional-ökonomische Konnotation - die "Ökonomisierung des Metropolendiskurses" (Blotevogel 2000: 146) - an. Metropolen werden eher in einen funktional-qualitativen Zusammenhang gestellt, als rein über demographisch-quantitative Merkmale definiert. Somit liefern Klassifikationen, die die Größe einer Stadt betonen, wie Auerbachs Rang-Größe-Regel (1913) oder die Phasen der Stadtentwicklung nach van den Berg, Drewett, Klaassen et al. (1982) bis hin zu neueren Publikationen über Mega-Cities von Bronger (2004), wenig Anhaltspunkte für Metropolen. Die Mindestgröße variiert und hängt von Fragestellung und/oder Untersuchungsraum ab. Ein Ansatz definiert sie mit mehr als einer Million, bis zu fünf Millionen Einwohnern und einer Mindesteinwohnerdichte von 2.000 Einwohnern pro Qua- dratkilometer mit monozentrischer Struktur (Bronger 2004: 24). Dennoch erscheint eine Mindestgröße eine notwendige Voraussetzung, da an solchen Standorten wichtige herausragende ökonomische, soziale, kulturelle und verkehrliche Funktionen zu lokalisieren sind und sich global vernetzen können.

\subsection{Metropolen als symbolische Orte der Inszenierung und kulturellen Strahlkraft}

Um die kulturelle Wirkung und gesellschaftliche Bedeutung von Metropolen haben sich überwiegend die Literatur- und Kulturwissenschaften bemüht, in denen dieser Stadttypus die höchste städtische Ausprägung und Dimension als "Stadt der Städte" (Kemper 2006: 16) einnimmt. Nach Reif (2006: 4) werden große Städte zu Metropolen, wenn sie in transmetropolitane Diskurse eingebunden sind und ein Selbstverständnis von Metropole geschaffen wird, das von der Gesellschaft anerkannt ist. Die viel gebrauchten Metaphern von Metropole als „Labor des Fortschritts“ (Reif 2006: 4) oder „Mythos Metropole“ (Fuchs/Moltmann/Prigge 1995) oder „Sinnbild an Urbanität, Gegenstand utopischer Entwicklung - und zugleich Symbol für Chaos, für Bedrohung, für die unmenschliche Welt von morgen, für Babylon" (Alter 1993: 13) beziehen sich darauf, dass Metropolen Symbolträger, Symbolproduzent und selbst Gegenstand von Bedeutungszuschreibungen sind.

Seit den 1990er-Jahren zeigt sich eine kulturwissenschaftliche Strömung in der Stadt- und Metropolenforschung, die die Aufmerksamkeit auf eine kulturelle und symbolische Bestimmung von Stadt lenkt (King 1991; Featherstone/Lash 1999). Damit einher geht ein erweitertes Verständnis in der Stadtgeographie, Raum bzw. Stadt nicht ausschließlich aus einer territorialen Perspektive zu analysieren, sondern Raum bzw. Stadt als geographische Identifikationspunkte mit kulturellen und symbolischen Bedeutungen zu interpretieren (Featherstone/Lash 1999). Metropolen als symbolische Referenzorte nehmen dabei eine Vorbildfunktion ein, sind Wegweiser oder geben Orientierung für Gesellschaft und Kultur (Häußermann 2000: 75; Mieg 2012b: 12). Im Zentrum der symbolischen Konnotation stehen die Einmaligkeit, die Ausstrahlung und das Wesen einer Stadt und weniger die Vergleichbarkeit von Städten. New York steht wie keine andere Stadt auf der Welt für Kosmopolitanität, Paris ist Sinnbild für Luxus und Liebe, Berlin verkörpert wie keine andere Stadt die deutsche Geschichte nach außen. Metropolen sind daher Orte, die im Kopf entstehen und als Elemente der mentalen Geographie 
bezeichnet werden (Häußermann 2000: 75). Sie haben eine eigene, unverwechselbare und stadtspezifische Symbolkraft, in der beispielsweise bauliche Ensembles zum Symbolträger werden - der Eiffelturm ist mit Paris verknüpft, das Brandenburger Tor mit Berlin und die Freiheitsstatue mit New York. Metropolen haben hier gegenüber anderen Städten große Vorteile, denn ihr Image mit historischen Besonderheiten und stadträumlichen Qualitäten ist bereits diskursiv vorstrukturiert und in den Köpfen der Menschen präsent (Bollerey 2010: 116).

Die kulturell-symbolische Betonung von Metropolen spiegelt sich in dem Wechselverhältnis zwischen Ökonomie und Kultur wider (Lash/Urry 2002; Amin 2006; Steets 2008). Die amerikanische Stadtsoziologin Zukin hat die Prozesse dieses Wettbewerbs zwischen Städten mit dem Stichwort "Ökonomie der Symbole" beschrieben (Zukin 1996: 44); letztlich geht es um das Ziel der Wertsteigerung von Orten. Die kulturellen und symbolischen Prozesse lösen Städte aus der räumlich verankerten Welt heraus und stellen sie in eine imaginäre Geographie. Über symbolische Alleinstellungsmerkmale, die einen Zeichenwert (Ökonomie der Symbole) versprechen, wird nicht nur der praktische und funktionelle Nutzen bestimmt, sondern ein symbolischer Nutzen ein Prestigegewinn (Petrin/Knieling 2009: 305).

Architektonische Großprojekte bzw. städtebauliche Prestigeobjekte werden bewusst als wichtiges Marketinginstrument und Teil der Inszenierung im Wettbewerb um Aufmerksamkeit, politische und ökonomische Macht im interkommunalen Konkurrenzkampf der Städte eingesetzt (Zukin 2010; Grubbauer 2011a; Grubbauer 2011b). Die symbolische Komponente solch repräsentativer Globalisierungsarchitektur ist von zentraler Bedeutung, weil sie den Einfluss mächtiger Akteure sichtbar macht und dazu beiträgt, eine spezifische Vorstellung von worldly globe zu manifestieren. Andererseits hat jeder Raum bzw. jede Stadt eine eigene Geschichte und Persönlichkeit und muss sich im Wettbewerb gleichzeitig durch unverwechselbare regionsspezifische oder auch Flagship-Architekturen, die das Regionale mit dem Globalen verbinden, profilieren.

\subsection{Ableitung der Dimensionen Größe, Funktion und Symbolik}

Die Ausführungen zum Metropolenbegriff verdeutlichen, dass der Begriff innerhalb der Stadtforschung in unterschiedliche Konzepte eingebunden ist. Dies bestätigt die Mehrdimensionalität des besonderen Stadttypus Metropole. Im Kern beziehen sich die Disziplinen zum einen auf eine spezifische Mindestgröße und -dichte als Unterscheidungsmerkmale zu kleineren als auch größeren Städten. Dazu gehören auch bauliche urbane Strukturen, hohe Dichte sozialer Interaktionen oder ethnische Vielfalt. Zum anderen kennzeichnet eine Metropole hochrangige verkehrliche, kulturelle, politische und wissenschaftliche Funktionen und Infrastrukturen mit nationaler und internationaler Reichweite. Ein drittes wesentliches Merkmal ist eine spezifische metropolitane Symbolik, die von Metropolen ausgeht und in den ökonomischen Metropolen- und Weltstadtdiskursen kaum Beachtung findet, nicht zuletzt, weil sie schwer zu quantifizieren ist und nicht zur Vergleichbarkeit von Städten taugt. Metropolen sind Träger von Symbolen und rufen eine bestimmte Vorstellung oder Bilder in den Köpfen hervor. Sie sind zentrale Orte der Auseinandersetzung um Repräsentationen von Geschichte, Kultur, urbanem Lebensgefühl, städtischen Lebensweisen und Macht. Damit dienen sie als Vorbild oder Referenz für andere Städte, an denen gesellschaftliche Veränderungen früher und deutlich abgelesen werden können.

Im Folgenden geht es darum zu untersuchen, wie der Metropolenbegriff in der deutschen Alltagssprache verwendet wird und mit welchen semantischen Schlüsselbegriffen, Zuschreibungen, Raumbildern, strukturellen Eigenschaften und Funktionen Metropolen sowie die drei Dimensionen Größe, Funktion und Symbolik im Alltagsdiskurs belegt werden und ob sich Brüche zum wissenschaftlichen Diskurs finden. Zudem können daraus Schlüsse gezogen werden, welche Städte im Sprachdiskurs als Metropolen gelten. Die zwei größten deutschen Städte, Berlin und Hamburg, gelten als Referenz für den deutschen Stadttypus Metropole und dienen als Fallbeispiele dafür, wie sich die Außendarstellung, sei es im Hinblick auf ein metropolitanes wie auch stadt- bzw. lokalspezifisches Profil vor und nach 1990 in Deutschland verändert hat.

Die Forschungsfragen lauten:

- Welche metropolitanen Eigenschaften und Zuschreibungen im Hinblick auf die Dimensionen Größe, Funktion und Symbolik kennzeichnen den Begriff Metropole im alltagsweltlichen Sprachgebrauch und welche Städte werden darin zu Metropolen erhoben?

- Spiegeln sich die drei Dimensionen Größe, Funktion und Symbolik in den Zuschreibungen der beiden größten deutschen Städte Berlin und Hamburg wider oder sind es stärker lokal- und stadtspezifische Eigenschaften, die das Bild der Städte prägen? Verändern sich im Zeitverlauf solche Zuschreibungen oder bleiben sie stabil? 


\section{Methodologische Grundannahmen}

Die Diskursanalyse, eine Methode zur Untersuchung übergeordneter Sinnzusammenhänge, sowie korpuslinguistische Ansätze sind Thema des folgenden Kapitels. Anwendung findet hier die Lexikometrie, ein Verfahren zur Analyse großer Textkorpora, deren methodische Grundlagen und Grundbegriffe im Folgenden erörtert werden.

\subsection{Diskursanalyse}

Die zunehmende Etablierung der Diskursanalyse bzw. die analytische Auseinandersetzung mit Diskursen ist nicht nur in den Literatur- und Medienwissenschaften, sondern auch in der Kulturgeographie (Mattissek 2008; Glasze/Mattissek 2009a) und der Humangeographie (Schmitt 2007; Golinski/Henn 2015) zu beobachten. Die Diskursforschung bzw. Diskursanalyse ist eine interdisziplinäre Debatte über die Konstruktion gesellschaftlicher Bedeutung des Symbolischen und eine Methode zur Untersuchung „übergeordneter" Sinnzusammenhänge (Keller 2011: 7). Vor allem die sozial- und geisteswissenschaftlichen Disziplinen sind in den diskurstheoretischen Theoriebildungen und Forschungen führend. Die Analysen über gesellschaftliche Bedeutungen von Wissen und symbolischen Bezugssystemen unterscheiden sich hinsichtlich ihrer theoretischen, empirischen und methodischen Herangehensweise. Während Diskurstheorien allgemeine theoretische Grundlagen für sprachliche Konstitutionen und Sinnstrukturen von Welt entwickeln, steht bei der Diskursanalyse das diskursive Feld kommunikativer Praktiken als gesellschaftliche Aktivität im Fokus (Keller 2011: 8).

Diskurse sind „kontingente und temporäre Fixierung von Bedeutungen" (Glasze 2008: 185), eine kollektiv verbindliche Wissensordnung in einem sozialen System, und unterliegen politischen und ökonomischen Machteffekten sowie zeitlichen Entwicklungen, die Bedeutungszuschreibungen und Sinnordnungen verändern. Es gilt daher, die Konstruktion von Begriffen und Objekten über einen zeitlichen Rahmen möglichst zu stabilisieren und herzustellen (Keller 2011: 7). Der Diskurs ist damit eine Verbindung von symbolischen Praktiken des Sprachund Zeichengebrauchs, materiellen Gegebenheiten und sozialen Institutionen (Glasze/Mattissek 2009b: 12).

Im Mittelpunkt der Diskursforschung steht damit die Analyse sprachlicher Sinngebungsprozesse, wie bestimmte Vorstellungen und Handlungslogiken herge- stellt werden, aber auch, wie sie sich verändern (Habermas 1981). Anders als Habermas argumentiert Foucault (1991). Für inn sind die zentralen Elemente des Diskurses gesellschaftliche Machtverhältnisse und Herrschaft, die auf die subjektiven Wissensordnungen einwirken. Aufgabe der Forscherin/des Forschers sei es, das Regelsystem zu rekonstruieren, indem beispielsweise die institutionell eingebundenen Regeln analysiert werden.

Mitte der 1980er-Jahre knüpfen die Politikwissenschaftler Laclau und Mouffe (1985) an Überlegungen des Diskursbegriffs von Foucault an und entwickeln die poststrukturalistische Diskurstheorie weiter. Sie betonen die Konstruktion von individuellen und kollektiven Identitäten. Danach existiert die Gesellschaft als Sinnordnung, in der materielle Objekte, Handlungen oder das Sprechen durch Bedeutungszuschreibungen hergestellt werden und alles zum Zeichenträger wird. Diskurse sind gesellschaftliche Praktiken, die Wirkungen von Sprache prägen, definieren und Wirklichkeiten konstruieren.

Die korpuslinguistische Diskursanalyse ist eine Akzentuierung der empirisch ausgerichteten Diskursanalyse. Sie erlaubt der Forscherin/dem Forscher, aus einer neuen Perspektive auf soziale Phänomene und sozialwissenschaftliche Fragestellungen Texte im Hinblick auf ihre sprachliche Konstitution mithilfe sprachwissenschaftlicher und kommunikationswissenschaftlicher Methoden zu untersuchen. Die Produkte sprachlicher Auseinandersetzung und Kommunikation, in denen sich bestimmte Symbole, Zeichen und Aussagen in einer bestimmten Anordnung finden, gilt es herauszufiltern und zu interpretieren (Scholz 2010: 86). Die korpuslinguistische Diskursanalyse hebt jedoch weniger auf die Fragen ab, wer und was diskursive Strukturen hervorbringt, sondern fragt danach, wie soziale, kulturelle, politische und ökonomische Strukturen und Identitäten diskursiv hergestellt werden (Glasze/Mattissek 2009b: 33). Sprachliche Zeichen können Bedeutungen über die Existenz anderer Begriffe erhalten. Somit kann die Sprachwissenschaft dafür genutzt werden, Diskurstheorie im Rahmen empirischer Forschungen zu operationalisieren (u. a. Mattissek 2008).

\subsection{Lexikometrie}

Ein Verfahren der Korpuslinguistik ist die Lexikometrie. Den Grundstein legte Ende der 1960er-Jahre Pêcheux (1969) mit der automatisierten Diskursanalyse, die aus Textstudien der Linguistik, der Statistik und der Informatik entsteht. Die "Lesemaschine“, die unabhängig von der Autorin/vom Autor die im Kontext enthaltenen Wort- 
formen analysieren kann, hat sich innerhalb der Korpuslinguistik als ein zentraler Ansatz zur Quantifizierung sprachlicher Strukturen etabliert (Lebart/Salem/Berry 1998).

Diese computergestützte Methode kann aus einem großen Textkorpus sprachliche Strukturen vollständig und automatisch sichtbar machen sowie Differenzbeziehungen, also quantitative Beziehungen, zwischen lexikalischen Elementen innerhalb eines Textkorpus herausarbeiten (vgl. Mattissek 2008). Mit der Lexikometrie können Regelmäßigkeiten von Beziehungen herausgefiltert und die mit Signifikanzen verknüpften lexikalischen Elemente operationalisiert werden. Kollokationen oder Kookkurrenzen sind hierbei Indikatoren zur Anzeige von Verknüpfungen, also welche Wörter und Wortfolgen (N-Gramme) im Korpus mit einer gewissen Signifikanz verbunden sind. Diese Wörter treten überdurchschnittlich häufig miteinander auf und werden daher als Kollokationspartner bezeichnet (Scherer 2006: 46; Glasze 2008: 202 f.; Dzudzek/Glasze/Mattissek et al. 2009: 245). Es werden signifikante Elemente (Kollokate) in direkter Umgebung des Schlüsselwortes ermittelt, z. B. zehn Wörter vor und hinter dem Schlüsselwort. Die Signifikanz drückt ein Wahrscheinlichkeitsmaß für das Auftreten eines Wortes im Verhältnis zu einem anderen Wort aus. Die Methodik kann daher angewendet werden, um den Metropolenbegriff in seinem Bedeutungsgehalt zu rekonstruieren, indem Zuschreibungen wiederholend im Zusammenhang mit dem Begriff Metropole auftreten. Das Verfahren der Erkenntnisgewinnung orientiert sich an einem induktiven Vorgehen, indem von Beobachtungen auf charakteristische Zusammenhänge geschlossen wird.

Die Methodik unterliegt in den Sozialwissenschaften dem Vorwurf, dass ausschließlich quantitative Strukturen gemessen und qualitative Aspekte vernachlässigt werden. Die Grenzen dieser Verfahren treten in dem Moment auf, in dem Rückschlüsse auf Kausalitäten, Temporalität oder Gegensätze gezogen werden sollen, die mit quantitativen Verfahren nicht analysiert werden können. Zum Beispiel können Verneinungen - keine Metropole - erst durch die Rückkopplung mit den Textstellen offengelegt werden. Ebenfalls kann ungesagtes implizites Wissen nicht erfasst werden. Den Grenzen dieser Verfahren stehen dennoch große Vorteile gegenüber. Die Methode bietet für die nachstehende Analyse die Möglichkeit, sehr große Textmengen zu erfassen, die auf Regelmäßigkeiten und Muster untersucht werden und allein durch Lesen nicht erfasst werden können. Außerdem können über eine induktive Herangehensweise diskursive Strukturen in zeitlicher Abfolge her- ausgearbeitet werden, die zum einen Unterschiede und Brüche innerhalb des Diskurses aufzeigen und zum anderen Bedeutungen durch regelmäßige Verknüpfungen herausarbeiten (vgl. Dzudzek/Glasze/Mattissek et al. 2009).

\subsection{Untersuchungskorpus}

Voraussetzung für die Durchführung der Analyse ist ein ,geschlossener', möglichst großer sowie dem gleichen Genre angehöriges digitales Textkorpus. Die Datenbank des "Instituts für deutsche Sprache" in Mannheim ist die größte elektronische Sammlung deutschsprachiger Texte - Zeitungstexte, belletristische, wissenschaftliche und populärwissenschaftliche Texte (Belica/Steyer 2006). Die Datenbank ermöglicht es, einzelne Zeitschriften und Zeitungen zu einem benutzerdefinierten Korpus für verschiedene Jahrgänge zusammenzustellen und mit dem Analysetool COSMAS (Corpus Search, Management and Analysis System) gezielte Konkordanz- und Kollokationsanalysen durchzuführen. Für die Untersuchung wird ein Korpus aus führenden sowie überregionalen deutschen Zeitschriften und Zeitungen (DIE ZEIT, Der Spiegel, Stern, Süddeutsche Zeitung und Focus) für den Zeitraum 1950 bis 2018 zusammengestellt, wobei nur Der Spiegel und DIE ZEIT in der Datenbank bis in die 1950er-Jahre zurückreichen (vgl. Tabelle 1). Sie gelten als Leitmedien und als repräsentativ für gesellschaftliche Kommunikationsprozesse, weil sie einen Zugang zu einem breiten Spektrum des öffentlichen Diskurses ermöglichen sowie unterschiedliche politische sowie gesellschaftliche Positionen der Diskursakteure gewährleisten (Reisigl/Ziem 2014: 92). Einschränkungen sind hinsichtlich des Sitzes der Redaktionen in manchen Untersuchungsstädten einzuräumen (z. B. Der Spiegel in Hamburg) und dass dasTextkorpus lediglich einen kleinen inhaltlichen und zeitlichen Ausschnitt der Wirklichkeit darstellt.

\section{Konstruktion des Begriffs in der Sprachpraxis}

In einer quantitativen Textanalyse wird der Metropolenbegriff in seinem Bedeutungsgehalt rekonstruiert, indem strukturelle, funktionale sowie symbolische Zuschreibungen, aber auch geographische Bezüge herausgearbeitet werden. Darüber hinaus werden die metropolitanen und die stadtspezifischen Zuschreibungen für die beiden 
Tabelle 1: Untersuchungseinheiten und Zeiträume der überregionalen Zeitschriften und Zeitungen ${ }^{4}$

\begin{tabular}{lll}
\hline & Zeitraum & $\begin{array}{l}\text { Treffer zu } \\
\text { Metropole }\end{array}$ \\
\hline Der Spiegel & $1950-2018 / J u n i$ & 2.966 \\
DIE ZEIT & $1953-2018 / J u n i$ & 4.083 \\
Focus & $2000-2018 / J u n i$ & 781 \\
Stern & $1996-2018 / J u n i$ & 728 \\
Süddeutsche Zeitung & 1992-2018/Juni & 8.924 \\
\hline
\end{tabular}

Datenquelle: CORPORA II-Datenbank des Instituts für deutsche Sprache, Mannheim

deutschen Großstädte Berlin und Hamburg in zeitlicher Entwicklung analysiert.

\subsection{Kollokationsprofil Metropole}

Zwischen 1950 und 2018 lassen sich insgesamt 17.482 Treffer zum Suchbegriff Metropole in 15.215 Texten ausfindig machen (Stand März 2019). Abbildung 2 zeigt das semantische Begriffsfeld zum Metropolenbegriff mit den Kollokationen innerhalb des Printmedien-Textkorpus, die zehn Wörter, die vor und hinter "Metropole" sowie innerhalb eines Satzes stehen und besonders häufig sowie mit einer hohen Signifikanz (Wahrscheinlichkeitsmaß für das Auftreten eines Wortes im Verhältnis zu einem anderen Wort) auftreten. Dabei werden die Begriffe in konzentrischen Ringen von außen nach innen anhand ihrer Signifikanz (10er Logarithmus) und der Häufigkeit des Auftretens (Größe der Schrift entspricht der Häufigkeiten) geordnet.

Die aus der theoretisch-konzeptionellen Diskussion abgeleiteten Dimensionen Größe, Funktion und Symbolik dienen im Folgenden als Systematisierungsansatz, an dem die Begriffe entlang gruppiert werden. Innerhalb der Dimensionen können sie durch die Funktionen Kultur, Politik, Verkehr, Wirtschaft und Wohnen klassifiziert werden. Die symbolische Dimension hat starke Bezüge zu stadtsoziologischen und kulturwissenschaftlichen Paradigmen, die Metropole mit den Bereichen Ausstrahlung, Fortschritt, Macht, Urbanität und Vielfalt

4 Es können nur Texte berücksichtigt werden, die innerhalb des COSMAS (Corpus Search, Management and Analysis System) zur Verfügung stehen. Daher kann z. B. die Süddeutsche Zeitung erst ab 1992 mit in die Analyse integriert oder andere Zeitungen, wie die Frankfurter Allgemeine Zeitung, nicht berücksichtigt werden. beschreiben und als Kategorien für die Einordnung der Dimension Symbolik dienen. Die Zuordnung der Begriffe zu den Gruppen erfolgt so objektiv wie möglich, dennoch hängt die Einteilung der Begriffe vom subjektiven Verständnis ab. Es ist daher möglich, dass die intersubjektiv abgestimmte Einordnung der Begriffe nicht von allen geteilt wird. Geographische Bezüge, also welche Städte und Regionen auf Metropolen verweisen, z. B. "Metropole und New York", werden in Kapitel 4.2 näher betrachtet.

Die charakteristischen Verweisstrukturen der Printmedienanalyse fächern ein mehrdimensionales Bild auf. Das Sprachprofil Metropole verweist auf Begriffe, die unmittelbar mit der Größe einer Stadt oder mit stadtstrukturellen Eigenschaften im Zusammenhang stehen. Begriffe wie Stadt, Welt, Großstadt, Zentrum, Million, groß verdeutlichen die Größendimension speziell zu den kontrastierenden Begriffen Provinz, provinziell, Dorf, Peripherie. Die funktionale Dimension hebt auf herausragende Funktionen, z. B. politische Machtfunktion (Hauptstadt, Regierung), ökonomische Funktionen (Wirtschaft, Finanz, Industrie, Hafen) oder verkehrliche Gatewayfunktionen (Verkehr, Bahn, Flug) ab. Leben, bewohnen, Bewohner, Wohnung sowie wohnen spiegeln Metropolen als Wohnort wider. Die kulturellen Begriffe verweisen einerseits auf die Hochkultur (Kunst, Theater), andererseits auf alltagskulturelle Konnotationen, z. B. sportliche Veranstaltungen (Olympia) oder den Städtetourismus (Hotel, Tourist, Reise, reisen). Die symbolische Dimension spannt ein Begriffsfeld mit zentralen Merkmalen der Urbanitätstheorie auf (urban, Moloch, lebendig, hektisch, Hochhaus, Wolkenkratzer). Damit einher gehen auch die Begriffe Wandel, wachsen, boomen, multi oder aufstreben, die die Vielfalt bzw. Dynamik repräsentieren. Aus der Genese des Metropolenbegriffs wissen wir, dass Metropolen auch immer Orte der Macht waren und bis heute sind, die durch die Begriffe erobern, zerstören, Terror, Reich repräsentiert werden. Die Ausstrahlung einer Metropole kann als metropolitane Zuschreibung interpretiert werden, die überwiegend mit positiven Eigenschaften assoziiert wird. Ruf, Image, blühen, strahlend, locken, kreativ oder glitzern verweisen auf semantische und symbolische Ausstrahlung und Wirkung dieses einzigartigen Stadttypus.

\subsection{Geographische Bezüge}

Metropolen werden nicht allein aufgrund statistischer Größenkennziffern (vgl. Kapitel 2.4) zur Metropole. Gesellschaftliche Aushandlungsprozesse, z. B. in den 


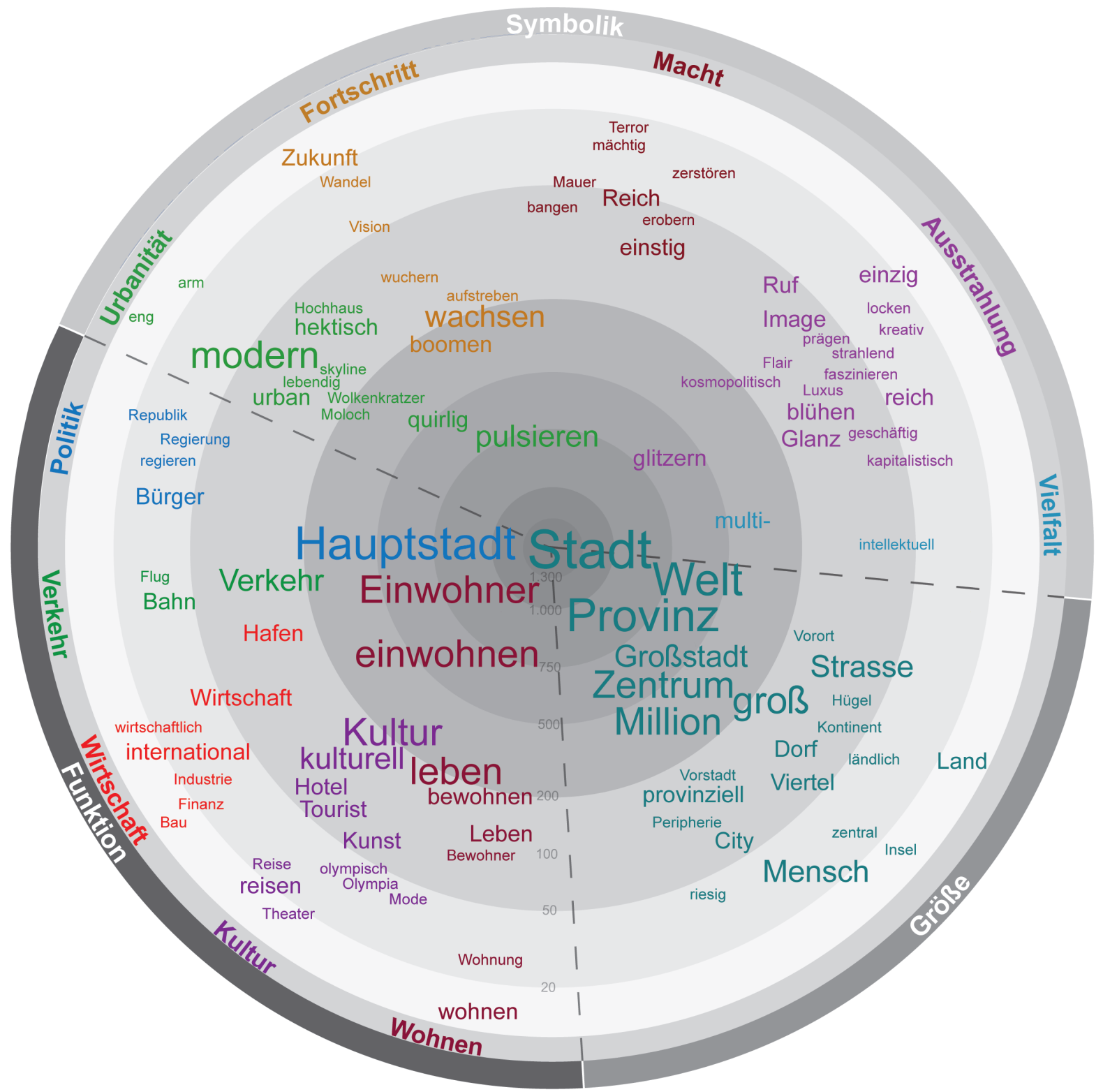

Abbildung 2: Kollokationsprofil Metropole

Datenquelle: CORPORA II-Datenbank des Instituts für deutsche Sprache

Anmerkung: Je weiter innen die Begriffe liegen, desto signifikanter sind sie. Die Größe der Begriffe entspricht der Häufigkeit ihres Vorkommens im Textkorpus.

Medien, belegen Städte mit metropolitanen Bildern und Zuschreibungen. Im Folgenden werden die geographischen Bezüge in der Nähe zur Metropole herausgearbeitet, die als Indikatoren dienen, welche Städte bzw. geographische Einheiten besonders häufig im Zusammenhang mit dem Begriff Metropole genannt werden und damit als Metropole bezeichnet werden können. Dabei muss berücksichtigt werden, dass es sich um deutsche Medien handelt, in denen deutsche Städte und Regionen signifikant höher und häufiger im Textkorpus auftreten als ausländische Städte und Regionen.
Abbildung 3 zeigt für die vier Untersuchungszeiträume 1950-1970, 1971-1990, 1991-2010, 2011-2018 die Städte oder Regionen, die vermehrt mit dem Begriff Metropole in Verbindung standen. Die Städte New York und Paris werden über alle vier Zeiträume hinweg wiederholt innerhalb des Kommunikationsprozesses als Metropolen genannt. Wurde bis 1970 die Stadt Bonn häufig im Zusammenhang mit Metropole genannt, nicht zuletzt aufgrund ihrer Funktion als Regierungssitz, sind es ab 1971 die Städte Berlin und Hamburg. Geographische Attribute wie amerikanische, bayerische, britische, fran- 
zösische, lombardische, mexikanische, niedersächsische, rheinische oder sowjetische Metropole verweisen auf bestimmte Städte der jeweiligen Länder oder Regionen, können jedoch nicht eindeutig einer Stadt zugeschrieben werden (z. B. „rheinische Metropole" = Köln, Bonn oder Düsseldorf).

Sind es bis 1990 vor allem deutsche (Bayern, Bonn, niedersächsisch, rheinisch), europäische (französisch, London, Belgrad) oder amerikanische (New York, Los Angeles, mexikanisch) Städte und Regionen, so treten ab den 1990er-Jahren auch vermehrt prosperierende asiatische Städte (Delhi, Chongping, Istanbul, Mumbai, Shanghai) auf. Politische Ereignisse wie Kriege in Syrien (Aleppo) oder im Irak (Mossul), Naturkatastrophen wie der Hurrikan Katrina in New Orleans, aber auch Sportereignisse wie die Fußball-WM 2014 oder die Olympischen Spiele (brasilianisch, Sao Paulo) lassen Städte zu bestimmten Zeitpunkten Gegenstand des Diskurses werden, die nicht zuletzt auch durch das Genre der Zeitschriften und Zeitungen beeinflusst werden. Insbesondere außen- und innenpolitische Themen werden dann in diesen Zeitungen vermehrt angesprochen.

\subsection{Kollokationsprofile Berlin und Hamburg}

Berlin und Hamburg sind nicht nur die beiden bevölkerungsreichsten Städte in Deutschland, sie sind zudem auch die beiden Städte, die über einen langen Zeitraum immer wieder in einem inhaltlichen Zusammenhang mit Metropole genannt und damit als deutsche Metropolen wahrgenommen werden (vgl. Abbildung 3). Im Folgenden werden daher die Sprachprofile vor 1989 und ab 1990 miteinander in Beziehung gesetzt. Das scheint insbesondere für Berlin sehr interessant, da es hier durch den Mauerfall 1989 und die Wiedervereinigung 1990 starke innenpolitische, soziale und wirtschaftliche Umbrüche gegeben hat, die sich in den Zuschreibungen an Berlin widerspiegeln müssten.

Die beiden Sprachprofile für Berlin decken spannende funktionale, aber auch symbolische Verweise auf. Die Dimensionen Größe und Funktion im unteren Bereich der Ringabbildung für die beiden Städte sind als allgemeine Zuschreibungen für Metropolen wiederzufinden. Die Begriffe innerhalb der Bereiche Kultur, Wirtschaft, Verkehr, Politik und Wissenschaft verweisen nicht eindeutig auf eine spezielle Stadt, sondern sind als metropolitane Zuschreibungen und Eigenschaften zu interpretieren. Berlin nahm und nimmt bis heute eine wichtige politische Funktion ein, was sich in der großen

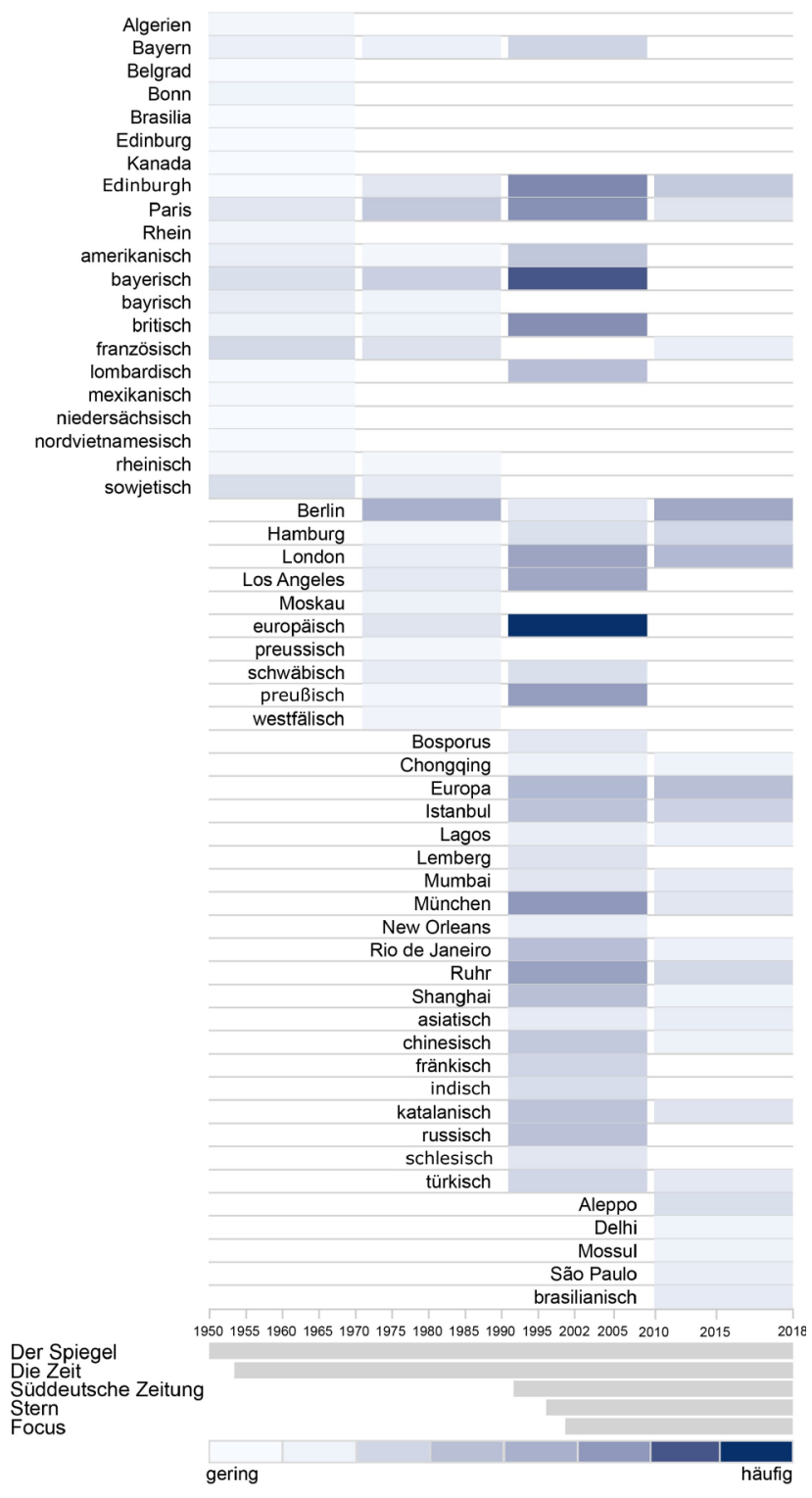

Abbildung 3: Geographische Bezugspunkte

Datenquelle: CORPORA II-Datenbank des Instituts für deutsche Sprache

Anmerkung: Je weiter innen die Begriffe liegen, desto signifikanter sind sie. Die Größe der Begriffe entspricht der Häufigkeit ihres Vorkommens im Textkorpus.

Zahl von Begriffen wie Bund, regieren, Senat, Botschafter, Koalition zeigt, aber auch im Bereich Politik, Wissenschaft und Forschung mit den Kollokationen Universität, Student, Institut Wirtschaft, Akademie, Kongress. Dagegen ist bis 1989 der Wirtschaftsbereich eher unterrepräsentiert. Lediglich Berlins Medienfunktion (Funk, Intendant) heben sich heraus. Bis heute sind die klassischen metropolitanen Wirtschaftsbereiche im Management, in Finanz- und Bankwesen oder Unternehmensund Rechtsberatung im Verhältnis zu den Bereichen 
Politik und Kultur unverhältnismäßig gering. Lediglich die Kollokationen Bank, international, Immobilien heben die ökonomische Bedeutung von Berlin als Finanzplatz heraus. Die verkehrliche Erreichbarkeit dieser Orte ist dabei ein entscheidender Faktor, global agieren zu können. Flug, Bahn, Flughafen verweisen unmittelbar auf die Funktion als Gateway, Theater, Kunst, Kultur und Museen auf Berlins Kulturfunktion.

Die lokal- bzw. stadtspezifischen Symboliken von Berlin und Hamburg stellt der obere Bereich der Ringabbildungen dar. Berlins lokale Außenwahrnehmung lehnt sich stark an die Geschichte an. Der Zweite Weltkrieg (Gestapo, Nazi, alliieren, Hitler, nachkriegs, Kriegsende, 1933, 1945), die Nachkriegszeit, der Kalte Krieg und die geteilte Stadt umgeben von DDR-Staatsgebiet (West, westdeutsch, Sowjet, DDR, westlich, Kennedy, Adenauer, amerikanisch) prägen Berlins symbolisches Profil bis 1989 (vgl. Abbildung 4). Nach dem Mauerfall und der Wiedervereinigung erlangt Berlin erneut Hauptstadtstatus. Der Einfluss der Medien ist insofern entscheidend, als Berlin seitdem ein starkes mediales Interesse erfährt, welches sich in der Anzahl der Kollokationen und der inhaltlichen Ausgestaltung des Semantikprofils widerspiegelt. Berlins politische Macht- und Kontrollfunktion als Bundeshauptstadt mit Regierungssitz sowie Berlins Rolle als Stadtstaat zeigt sich auch in der Außendarstellung ab 1990 (vgl. Abbildung 5), z. B. mit den Begriffen Bundestag, Bundeskanzler, Merkel sowie den Politikerinnen und Politikern Schäuble, Gabriel, Diepgen, Sarrazin, Schröder, Eichel, Stoiber, Seehofer, Schily oder Künast.

Die Kulturökonomie (der Film Babylon Berlin, die Fashion Week, die Verlage Ullstein, Suhrkamp und Springer) oder Sportvereine (SCC, Tennis Borussia, Eisbär, Hertha, Alba) werden häufig im Zusammenhang mit Berlin verwendet, schon allein, weil sie Teil des Namens sind. Berlin ist die meistbesuchte Stadt Deutschlands und wird mit zahlreichen touristischen Destinationen und Sehenswürdigkeiten verbunden (z. B. Alexanderplatz, Potsdamer Platz, Spree, Kurfürstendamm, Reichstag). Sie verweisen auf die stadtspezifische Architektur von Bauten und Stadtteilen. Gleichzeitig signalisiert das neue Potsdamer-Platz-Quartier eine Global-City-Architektur.

Die Kollokationsprofile für Hamburg spannen ein diversifiziertes Spektrum auf, was nicht zuletzt auch auf Hamburg als Verlagsort für die Medien Der Spiegel, Stern und DIE ZEIT zurückzuführen ist. Die unterschiedlich starke Belegung zwischen den beiden Zeiträumen ist auf den geringeren Bestand der Zeitschriften und Zeitungen vor 1989 zurückzuführen.
Bis 1989 ist das funktionale Sprachprofil für Hamburg im unteren Bereich der Ringabbildungen durch hochrangige Funktionen aus ökonomischen (Bank, Geschäft, Handel, Industrie), politischen (SPD, Bund, Senat, Wahl) und kulturellen (Kunst, Theater, Oper, Fernsehen) Bereichen geprägt (vgl. Abbildung 6). Lediglich der Begriff Großstadt verweist auf die Größe. Die symbolischen Zuschreibungen verweisen einerseits auf das Drehkreuz im deutschen, aber auch internationalen Seehafenverkehr als auch auf den Verlags-, Musik- und Werbestandort. Bei genauerer Betrachtung hat sich das Kollokationsprofil für die Stadt Hamburg ab 1990 kaum verändert. Es findet sich ebenfalls eine große Anzahl an funktionalen und symbolischen Zuschreibungen aus Politik, Kultur, Wirtschaft und Wissenschaft. Es zeigt sich eine gewisse Stabilität hochrangiger Funktionen als auch metropolitaner Symbolik für die Stadt Hamburg. Als Freie und Hansestadt Hamburg hat sie als Stadtstaat und Stadt überörtliche politische (SPD, Senat, Bundesland), wirtschaftliche (Handel, Vorstand, Immobilie, Mineralöl), verkehrliche (Flug, Flugzeug, fliegen) sowie wissenschaftliche Funktionen (Universität, Uni, Institut).

Die Begriffe Hanse, Hafen, Schiff aus dem ökonomischen und Rowohlt, Springer und Oetinger aus dem kulturell-ökonomischen Feld belegen Hamburg mit lokalen Zuschreibungen. In den 1980er-Jahren war die Fusion des Hamburger Nordwest-Kraftwerk in die Muttergesellschaft ein zentrales Thema in der Presse. (Helmut) Schmidt, Elbe, norddeutsch, hanseatisch, Altona, Pauli, Alster haben lokale Bezüge und verweisen direkt auf die Stadt Hamburg.

Hamburg wird nicht umsonst wegen seiner Hafenfunktion und des internationalen Flughafens „Deutschlands Tor zur Welt" genannt. Hafen, Schiff, Hapag (Transport- und Logistikunternehmen), Container, Blohm + Voss (Schiffswerft) oder Werft beziehen sich auf ökonomische Bereiche und prägen das Einzigartige, die Außendarstellung der Stadt. Darüber hinaus wird Hamburg als historisch geprägte Handelsstadt mit den Attributen Handel und Kaufmann als auch mit metropolitanen Wirtschaftstätigkeiten der Firmen $\mathrm{HSH}$ (Hamburg Commercial Bank AG), Airbus oder Mobilcom belegt. Hamburgs politische Symbolik wird insbesondere in der Zeit nach dem 11. September 2001 oft mit Terrorismus (Mounir al-Motassadeq, Atta Mohammed), mit Krawallen (Krawall) oder mit der linksorientierten Szene in Verbindung gebracht (Rote Flora). Dabei sind es auch die ehemaligen Bürgermeister (Roland) Schill, Ole von Beust, Olaf Scholz, die das politische Bild der Stadt prägen. 


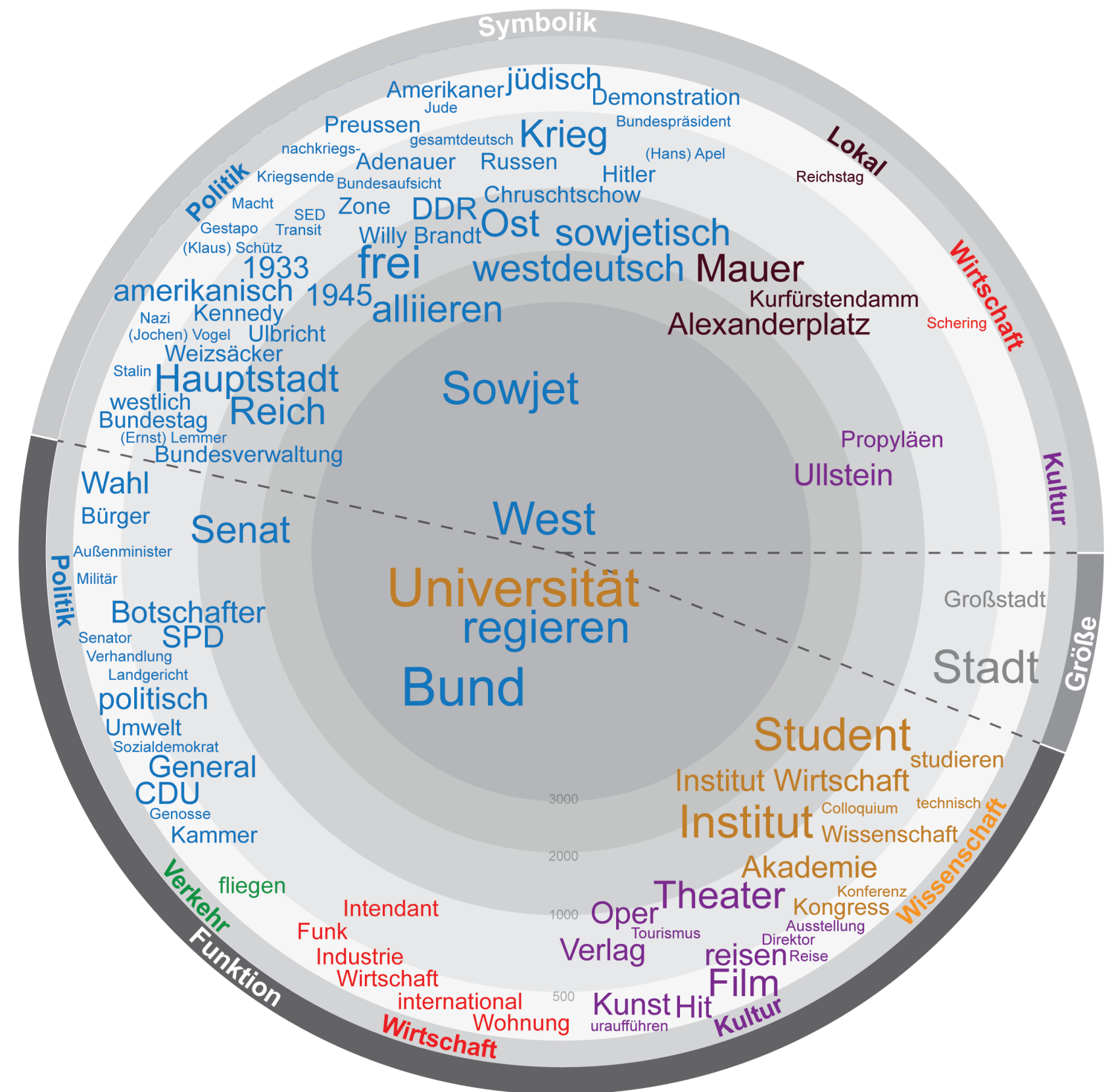

Abbildung 4: Kollokationsprofil Berlin 1950-1989

Datenquelle: CORPORA II-Datenbank des Instituts für deutsche Sprache

Anmerkung: Je weiter innen die Begriffe liegen, desto signifikanter sind sie. Die Größe der Begriffe entspricht der Häufigkeit ihres Vorkommens im Textkorpus.

Nautilus, Oetinger, Rowohlt beziehen sich auf den Verlagsstandort Hamburg, der neben Berlin und München zu den drei bedeutendsten Verlagsstandorten in Deutschland zählt (vgl. Abbildung 7). Einen großen Bereich umfasst die breit ausdifferenzierte kulturelle Branche. Hochkultur meets Szenekultur - das trifft sowohl für Berlin als auch für Hamburg zu und so stehen die hochkulturellen Merkmale (Schauspiel, Theater, Schauspielhaus, Musical, Ballett) neben sportlichen Events (Freezers (ehemalige Eishockeymannschaft), HSV, UHC (Uhlenhorster Hockey-Club e.V.), german open, (Thomas) Haas oder Reeperbahn, (Sankt) Pauli. Hamburgs Sehenswürdigkeiten verweisen gleichzeitig auf eine stadtspezifische metropolitane Architektur 


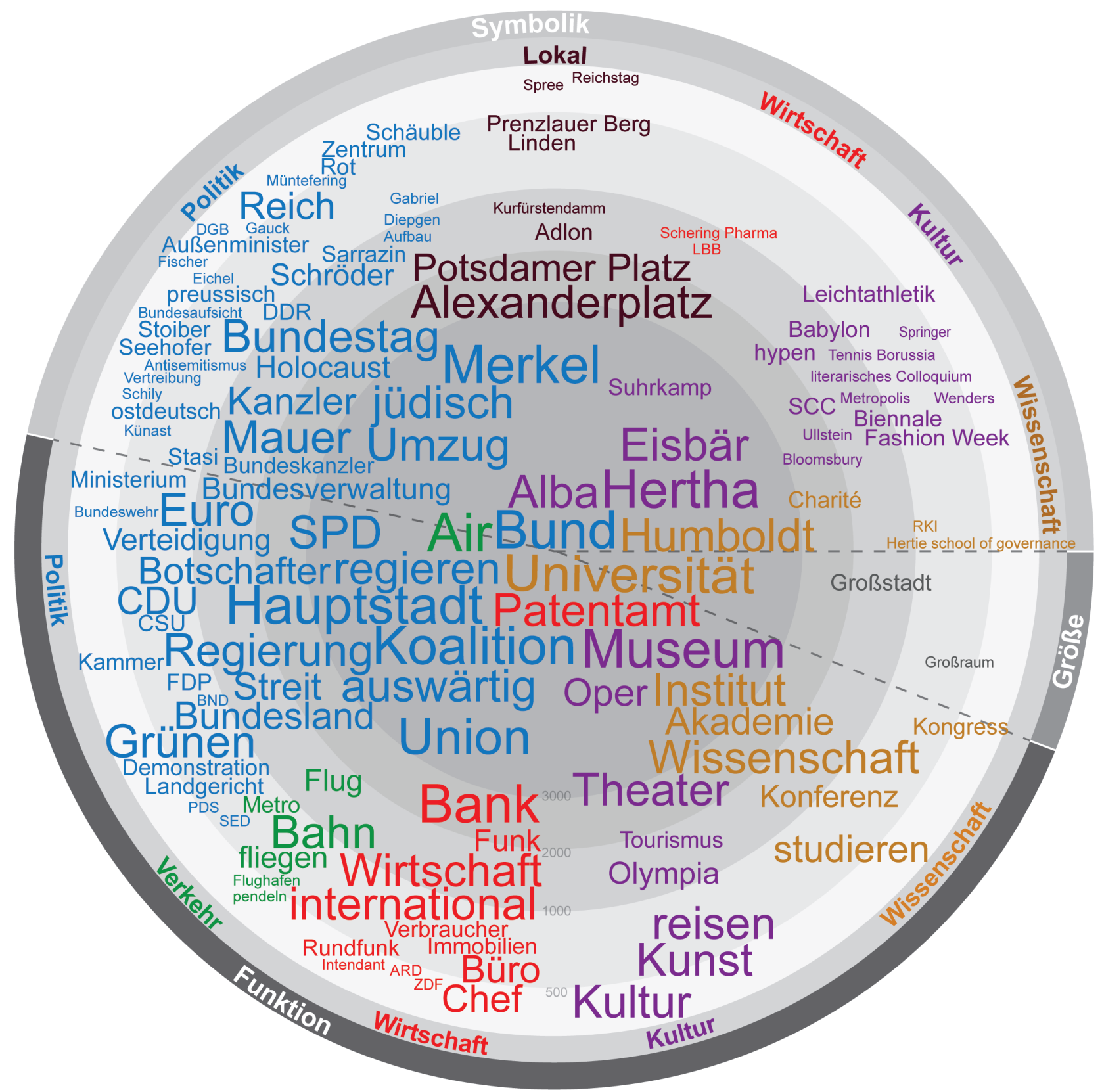

Abbildung 5: Kollokationsprofil Berlin 1990-2018

Datenquelle: CORPORA II-Datenbank des Instituts für deutsche Sprache

Anmerkung: Je weiter innen die Begriffe liegen, desto signifikanter sind sie. Die Größe der Begriffe entspricht der Häufigkeit ihres Vorkommens im Textkorpus.

sowie auf die Tourismusdestination Hamburg. Die Elbphilharmonie ist hierfür ein gutes Beispiel. In der Presse erlangte sie eine große Resonanz im positiven wie auch im negativen Sinne. Jedenfalls wird die Elbphilharmonie hochsignifikant häufig im selben Atemzug mit Hamburg genannt.

Aus den Kollokationsanalysen für Berlin und Hamburg lässt sich herauslesen, dass symbolische
Zuschreibungen an Metropolen, die auf lokal- bzw. stadtspezifische Eigenschaften verweisen, mindestens genauso bedeutend sind wie funktionale Zuschreibungen. Internationale hochrangige Funktionen, mit denen sich Metropolen von anderen Städten unterscheiden, können ebenfalls im Alltagsdiskurs für Berlin und Hamburg, wenn auch in unterschiedlicher Form, für beide Zeiträume nachgewiesen werden. Die lokalen 


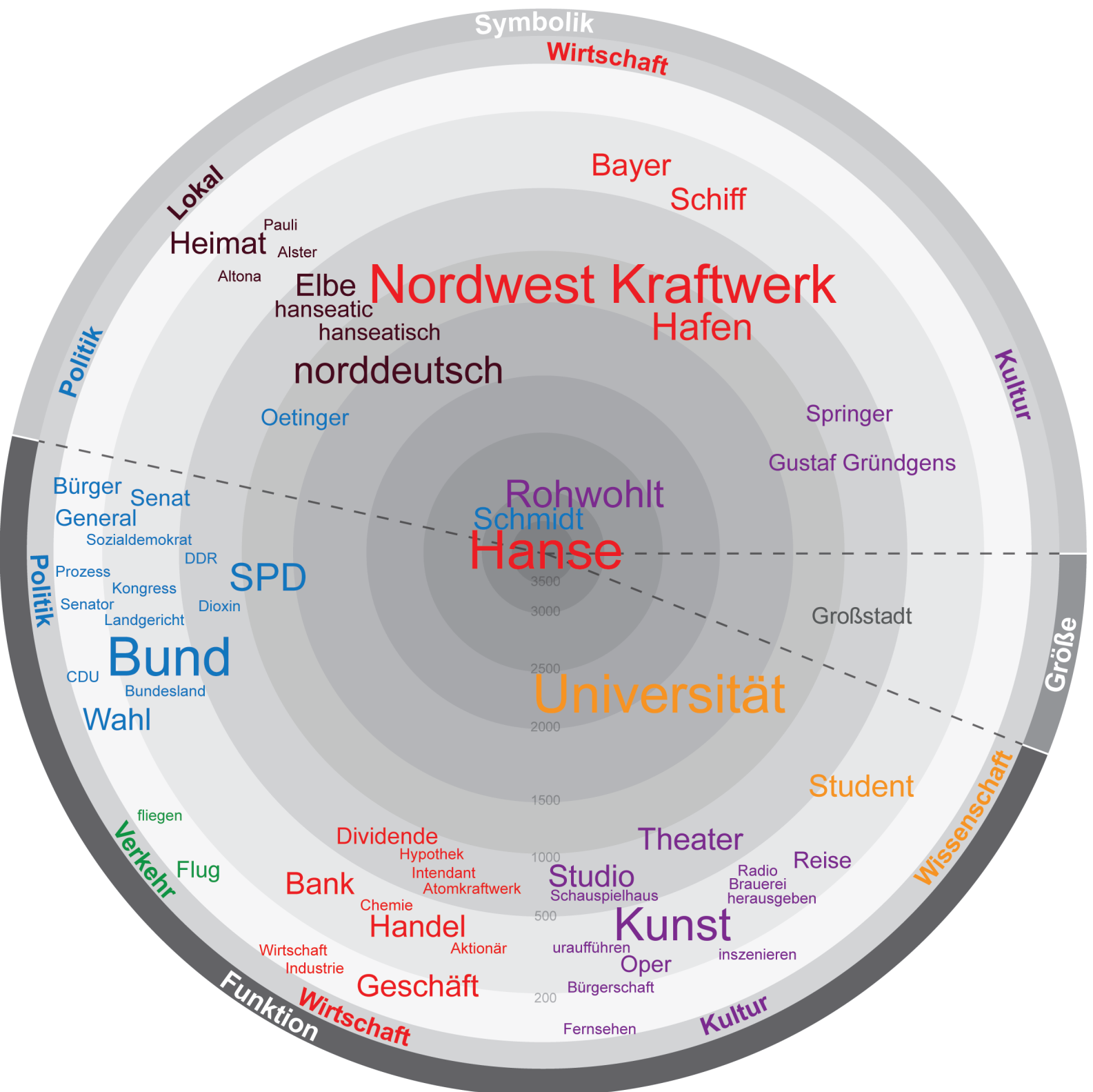

Abbildung 6: Kollokationsprofil Hamburg 1950-1989

Datenquelle: CORPORA II-Datenbank des Instituts für deutsche Sprache

Anmerkung: Je weiter innen die Begriffe liegen, desto signifikanter sind sie. Die Größe der Begriffe entspricht der Häufigkeit ihres Vorkommens im Textkorpus.

Zuschreibungen an Berlin und Hamburg lassen sich nicht eindeutig in den Kategorien Urbanität, Fortschritt, Macht, Ausstrahlung oder Vielfalt der metropolitanen Symbolik wiederfinden, stattdessen weisen sie indirekt auf diese Attribute hin, z. B. Potsdamer Platz auf Hochhaus oder Skyline, das Adlon auf Luxus. Das führt zu der Schlussfolgerung, dass der Alltagsdiskurs um den Metropolenbegriff sehr eng mit dem Metropolendiskurs der Stadtforschung verzahnt ist.

\section{Fazit}

Die Annäherung an den Metropolenbegriff legt die begriffliche Mehrdimensionalität dar: die drei Dimensionen (1) Mindestgröße und -dichte, (2) ein hochrangiges Funktionsspektrum mit nationaler und internationaler Reichweite sowie (3) eine metropolitane Symbolik. Sie dienen als Grundgerüst für Systematik und Interpretation der quantitativ angelegten Printmedienanalyse. 


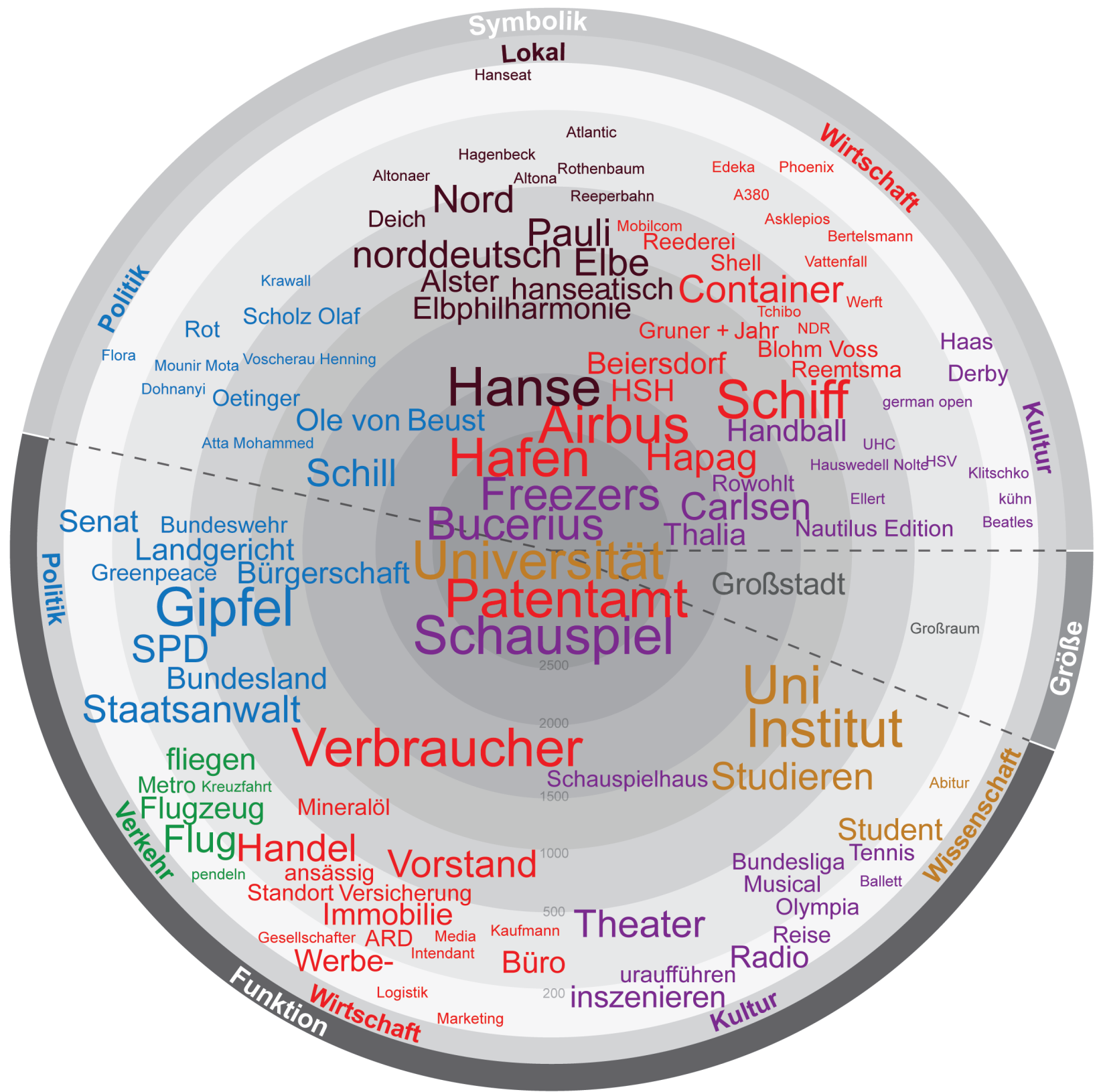

Abbildung 7: Kollokationsprofil Hamburg 1990-2018

Datenquelle: CORPORA II-Datenbank des Instituts für deutsche Sprache

Anmerkung: Je weiter innen die Begriffe liegen, desto signifikanter sind sie. Die Größe der Begriffe entspricht der Häufigkeit ihres Vorkommens im Textkorpus.

Die herausgearbeiteten Begriffe im alltagsweltlichen Sprachgebrauch im Kontext von Metropole, Berlin und Hamburg sind als Bedeutungszuschreibungen bzw. als Sprechen über die Stadt und nicht als Selbstdarstellung oder Selbstinszenierung der Städte zu interpretieren. Bei der Festigung von Raumbildern spielen die Printmedien eine tragende Rolle, die Wirklichkeiten vorstrukturieren oder Sinndeutungen an den Raum in den Köpfen der Menschen hervorrufen. Die Bedeutungszuschreibungen unterliegen dabei politischen, sozialen sowie kulturellen Aushandlungen und spiegeln gesellschaftliche Machtverteilungen und Organisationsprinzipien wider. Das zeigt sich im Speziellen in den Verschiebungen der geographischen Bezüge auf der Zeitachse (vgl. Abbildung 3). Dabei reproduzieren die Printmedien einerseits einen metropolitanen Stadttypus, losgelöst von bestimmten Orten mit hochrangigen Funktionen, und andererseits stadtspezifische Eigenschaften, die das Eigene und 
damit den Charakter der Stadt betonen und sie von anderen Städten unterscheiden. Die immer stärkere Ausdifferenzierung und globale Verbreitung globaler Konsum- und Kulturgüter hebt gleichzeitig in einer weltweiten Homogenisierung auf die Frage nach Lokalität ab. Für dieses Verhältnis haben Robertson und Winter (2000) und Swyngedouw (2004) den Begriff „Glokalisierung" geprägt, mit dem mehr als nur die Betonung des Lokalen im Gegensatz zum Fremden gemeint ist und auf die Verankerung des "Globalen im Lokalen“ sowie des „Lokalen im Globalen“ zielt. Dieses Gegensatzpaar des Globalen und Lokalen ist genau das, was eine Metropole ausmacht und sie von anderen Städten unterscheidet. Der Hauptstadt werden zentrale globale Funktionen politischer sowie politiknaher, hochkultureller und kulturökonomischer Organisationen sowie wissenschaftlicher Einrichtungen zugeschrieben. Demgegenüber zeigt das metropolitane Profil Hamburgs ein ausgeglichenes Spektrum metropolitaner Funktionen. Das Lokale verweist auf stadtspezifische Besonderheiten. Berlins symbolisches Bild ist stark durch den politisch-geschichtlichen Werdegang, kulturelle, aber auch städtebauliche Symbole (Potsdamer Platz, Kurfürstendamm, Reichstag) geprägt. Ähnlich verhält es sich mit Hamburg: Die Bilder der Stadt sind stark von geographischen Bezügen des Nordens und der Hafenfunktion beeinflusst.

Die reproduzierten Bilder beider Städte sind Bestandteil der Stadtimages und gelten als zentrale Standortfaktoren im Wettbewerb um Touristen, neue Einwohnerinnen/Einwohner, qualifizierte Arbeitskräfte und Investoren. Neben funktionalen Wettbewerbsvorteilen sind es daher symbolische Vorteile bzw. weiche Standortfaktoren, die bei deren Stadt- und Standortwahl entscheidenden Einfluss haben. Der Wunsch nach positiver Aufmerksamkeit und einem attraktiven Stadtimage wird seit einigen Jahren auch von Seiten der Stadtplanung und des Stadtmarketings erkannt, wenn sich große Städte selbst nicht nur im Marketing als Metropole bezeichnen, sondern über die Selbstbeschreibung peu à peu auch ein Selbstverständnis als Metropole entwickeln, sei es nur als Anspruch oder sei es als täglich gelebte Realität im Bewusstsein der Bevölkerung. City branding (Roost 2008), place branding (Stöber 2007; Müller 2013), städtebauliche Großprojekte (BMVBS 2011) oder Leitbilder sollen dazu dienen, Identifikation nach innen und außen zu generieren. Wenn wir an Stadtbilder denken, denken wir allerdings in erster Linie an architektonische Bauwerke, die als Symbole für die Stadt gelten. Hamburgs Elbphilharmonie war noch nicht fertig, dennoch war das neue Wahrzeichen der Stadt schon in den Köpfen verankert. Anders ist es bei sprachlichen Zuschreibungen wie Leitbildern. Die Leitbilder "Wachsende Metropole“ Hamburg und "be Berlin" bzw. das neue Leitbild „Berlin bleibt anders" sollen identitätsstiftende Funktion haben, dennoch geht es immer verstärkt auch um die Stadt als Marke im Wettbewerb um Unternehmen und Haushalte.

Darum sind Großstädte nicht per se Metropolen. Städte werden erst in gesellschaftlichen Kommunikationsprozessen und Aushandlungen zu Metropolen erhoben, wenn sie mit visuellen Images, Symbolen, Bildern und Erinnerungen in der Werbung, im Film als Kulisse, in der Kunst als Objekt oder im Stadtmarketing als Marke mit metropolitanen Bildern und Zuschreibungen belegt werden und damit ein Selbstverständnis von Metropole geschaffen wird, das von der Gesellschaft anerkannt ist. Eine Metropole ist daher auch immer ein Repräsentations- und Bedeutungsraum und steht im Wirkungsfeld von Repräsentanz, Aufmerksamkeit, medialen Inszenierungen und ökonomischer Inwertsetzung.

\section{Literatur}

Alter, P. (1993): Einleitung. In: Alter, P. (Hrsg.): Im Banne der Metropolen. Berlin und London in den zwanziger Jahren. Göttingen, 7-22. = Veröffentlichungen des Deutschen Historischen Instituts London 29.

Amin, A. (2006): Kulturelle Ökonomie und Stadt. In: Berndt, C.; Glückler, J. (Hrsg.): Denkanstöße zu einer anderen Geographie der Ökonomie. Bielefeld, 111-135.

Auerbach, F. (1913): Das Gesetz der Bevölkerungskonzentration. In: Petermanns Geographische Mitteilungen 59, 74-76.

BBSR - Bundesinstitut für Bau-, Stadt- und Raumforschung (Hrsg.) (2011): Metropolitan areas in Europe. Bonn. = BBSR OnlinePublikation 01/2011. https://www.bbsr.bund.de/BBSR/EN/ Publications/OnlinePublications/2011/DL_ON012011.pdf? blob=publicationFile\&v=2 (05.09.2019).

Beaverstock, J.; Smith, R.; Taylor, P. (1999): A roster of world cities In: Cities 16, 6, 445-458. doi: 10.1016/S0264-2751(99)00042-6

Belica, C.; Steyer, K. (2006): Korpusanalytische Zugänge zu sprachlichem Usus. In: Vachková, M. (Hrsg.): Beiträge zur bilingualen Lexikographie. Prag, 7-24.

Blotevogel, H. H. (2000): Gibt es in Deutschland Metropolen? Die Entwicklung des deutschen Städtesystems und das Raumordnungskonzept der „Europäischen Metropolregionen“. In: Matejovski, D. (Hrsg.): Metropolen. Laboratorien der Moderne. Frankfurt am Main, 139-167.

Blotevogel, H. H. (2002): Deutsche Metropolregionen in der Vernetzung. In: Informationen zur Raumentwicklung 6/7, 345351.

BMRBS - Bundesministerium für Raumordnung, Bauwesen und Städtebau (1995): Raumordnungspolitischer Handlungsrahmen. Bonn.

BMVBS-Bundesministerium für Verkehr, Bau und Stadtentwicklung (Hrsg.) (2011): Stadtentwicklung und Image. Städtebauliche Großprojekte in Metropolräumen. Berlin. = Forschungen 150. 
Bollerey, F. (2010): Mythos Metropolis. Die Stadt als Sujet für Schriftsteller, Maler und Regisseure. Berlin.

Bonneville, M. (1994): Internationalization of non-capital cities in Europe: Aspects, processes and prospects. In: European Planning Studies 2, 3, 267-285. doi: 10.1080/09654319408720268

Bronger, D. (2004): Metropolen, Megastädte, Global Cities. Die Metropolisierung der Erde. Darmstadt.

Danielzyk, R. (2012): Der raumordnungspolitische Metropolendiskurs - Konstruktion von (neuen) Peripherien? In: disP - The Planning Review 48, 2, 27-33. doi: 10.1080/02513625.2012.721601

Derudder, B.; Taylor, P. (2016): Change in the World City Network, 2000-2012. In: The Professional Geographer 68, 4, 624-637. doi: 10.1080/00330124.2016.1157500

Dzudzek, I.; Glasze, G.; Mattissek, A.; Schirmel, H. (2009): Verfahren der lexikometrischen Analyse von Textkorpora. In: Glasze, G.; Mattissek, A. (Hrsg.): Handbuch Diskurs und Raum. Theorien und Methoden für die Humangeographie sowie die sozial- und kulturwissenschaftliche Raumforschung. Bielefeld, 233-260.

Engel, E.; Lambrecht, K. (1995): Hauptstadt - Residenz Residenzstadt - Metropole - Zentraler Ort. Probleme inrer Definition und Charakterisierung. In: Engel, E.; Lambrecht, K.; Nogossek, H. (Hrsg.): Metropolen im Wandel. Zentralität in Ostmitteleuropa an der Wende vom Mittelalter zur Neuzeit. Berlin, 11-32.

Featherstone, M.; Lash, S. (Hrsg.) (1999): Spaces of culture. City, nation, world. London.

Foucault, M. (1991): Die Ordnung des Diskurses. Frankfurt am Main.

Friedmann, J. (1986): The World City Hypothesis. In: Development and Change 17, 1, 69-83. doi: 10.1111/j.1467-7660.1986. tb00231.x

Fuchs, G.; Moltmann, B.; Prigge, W. (Hrsg.) (1995): Mythos Metropole. Frankfurt am Main.

Gerhard, U. (2004): Global Cities. Anmerkungen zu einem aktuellen Forschungsfeld. In: Geographische Rundschau 56, 4, 4-10.

Glasze, G. (2008): Vorschläge zur Operationalisierung der Diskurstheorie von Laclau und Mouffe in einer Triangulation von lexikometrischen und interpretativen Methoden. In: Historical Social Research 33, 1, 185-223. doi: 10.12759/ hsr.33.2008.1.185-223

Glasze, G.; Mattissek, A. (Hrsg.) (2009a): Handbuch Diskurs und Raum. Theorien und Methoden für die Humangeographie sowie die sozial- und kulturwissenschaftliche Raumforschung. Bielefeld.

Glasze, G.; Mattissek, A. (2009b): Diskursforschung in der Humangeographie: Konzeptionelle Grundfragen und empirische Operationalisierungen. In: Glasze, G.; Mattissek, A. (Hrsg.): Handbuch Diskurs und Raum. Theorien und Methoden für die Humangeographie sowie die sozial- und kulturwissenschaftliche Raumforschung. Bielefeld, 7-59.

Golinski, S.; Henn, S. (2015): Imperialisten, Spione oder Retter? Zur Charakterisierung von Direktinvestitionen aus Russland, Indien und China in deutschen Tageszeitungen. In: Zeitschrift für Wirtschaftsgeographie 59,1,1-19. doi: 10.1515/zfw.2015.0001

Grubbauer, M. (2011a): Globale Architektur und ihre Rolle im Städtewettbewerb. In: Parnreiter, C. (Hrsg.): Stadt und
Globalisierung. Hamburg, 45-65. = Hamburger Symposium Geographie 3.

Grubbauer, M. (2011b): Die vorgestellte Stadt. Globale Büroarchitektur, Stadtmarketing und politischer Wandel in Wien. Bielefeld.

Habermas, J. (1981): Theorie des kommunikativen Handelns. Frankfurt am Main.

Häußermann, H. (2000): Es muss nicht immer Metropole sein. In: Matejovski, D. (Hrsg.): Metropolen. Laboratorien der Moderne. Frankfurt am Main, 67-79.

Häußermann, H. (2011): Georg Simmel, der Stadtsoziologe. Zur Einführung. In: Mieg, H. A.; Sundsbø, A. O.; Bieniok, M. (Hrsg.): Georg Simmel und die aktuelle Stadtforschung. Wiesbaden, 15-27. doi: 10.1007/978-3-531-93132-6

Hall, P. (1966): The World Cities. London.

Heineberg, H. (2014): Stadtgeographie. Paderborn.

Helbrecht, I. (2013): Urbanität und Ruralität. In: Lossau, J.; Freytag, T.; Lippuner, R. (Hrsg.): Schlüsselbegriffe der Kultur- und Sozialgeographie. Stuttgart, 167-181.

Hose, M.; Levin, C. (2009): Der Geist braucht Metropolen Metropolen brauchen den Geist. In: Hose, M.; Levin, C. (Hrsg.): Metropolen des Geistes. Frankfurt am Main, 9-20.

Hoyler, M. (2004): London und Frankfurt als Weltstädte. Globale Dienstleistungszentren zwischen Kooperation und Wettbewerb. In: Geographische Rundschau 56, 4, 26-31.

Keller, R. (2011): Diskursforschung. Eine Einführung für SozialwissenschaftlerInnen. Wiesbaden. doi: 10.1007/978-3531-92085-6

Kemper, F.-J. (2006): Metropolen: Merkmale, Konzepte, Perspektiven. In: Schultz, H.-D. (Hrsg.): Metropolitanes \& Mediterranes. Beiträge aus der Humangeographie. Berlin, 1-20.

King, A. D. (Hrsg.) (1991): Culture, globalization and the worldsystem. Contemporary conditions for the representation of identity. Basingstoke.

Laclau, E.; Mouffe, C. (1985): Hegemony and socialist strategy: towards a radical democratic politics. London.

Lash, S.; Urry, J. (2002): Economies of signs and space. London.

Lebart, L.; Salem, A.; Berry, L. (1998): Exploring textual data. Dordrecht. $=$ Text, speech and language technology 4

Lees, A. (1984): The Metropolis and the Intellectual. In: Sutcliffe, A. (Hrsg.): Metropolis 1890-1940. London, 67-94.

Matejovski, D. (Hrsg.) (2000): Metropolen. Laboratorien der Moderne. Frankfurt am Main.

Mattissek, A. (2008): Die neoliberale Stadt. Diskursive Repräsentationen im Stadtmarketing deutscher Großstädte. Bielefeld.

Mieg, H. A. (2012a): Labore der Moderne - Schaltstellen der Globalisierung: Was Metropolen ausmacht. In: Althaus, M.; Göhler, G.; Schmalz-Jacobsen, C.; Walther, C. (Hrsg.): Medien, Macht und Metropolen. Politische Kommunikation in der Großstadt. Frankfurt am Main, 13-28.

Mieg, H. A. (2012b): Metropolen: Begriff und Wandel. In: Oberste, J. (Hrsg.): Metropolität in der Vormoderne. Konstruktionen urbaner Zentralität im Wandel. Regensburg, 11-33. = Forum Mittelalter-Studien 7.

Müller, A. (2013): „So kreativ wie Berlin, so erfolgreich wie Frankfurt“ - der symbolische Nutzen von Städten als Zielgröße einer imageorientierten Stadtmarkenführung. In: Raumforschung 
und Raumordnung 71, 2, 115-128. doi: 10.1007/s13147-0130236-9

Nitt-Drießelmann, D.; Wedemeier, J. (2017): HWWI/BerenbergStädteranking 2017: Die 30 größten Städte Deutschlands im Vergleich. Hamburg.

Pêcheux, M. (1969): Analyse automatique du discours. Paris.

Petrin, J.; Knieling, J. (2009): Das Bildversprechen der Metropolregion - Potenziale und Risiken einer bildmächtigen Raumkategorie. In: Knieling, J. (Hrsg.): Metropolregionen. Innovation, Wettbewerb, Handlungsfähigkeit. Hannover, 300322. = Forschungs- und Sitzungsberichte der ARL 231.

Reif, H. (2006): Metropolen. Geschichte, Begriffe, Methoden. Berlin. = CMS Working Paper Series 001-2006.

Reisigl, M.; Ziem, A. (2014): Diskursforschung in der Linguistik. In: Angermüller, J.; Nonhoff, M.; Herschinger, E.; Macgilchrist, F.; Reisigl, M.; Wedl, J.; Wrana, D.; Ziem, A. (Hrsg.): Diskursforschung. Ein interdisziplinäres Handbuch. Band 1: Theorien, Methodologien und Kontroversen. Bielefeld, 70-110.

Ritter, E.-H. (1998): Metropolen im supranationalen Wettbewerb. In: ARL - Akademie für Raumforschung und Landesplanung (Hrsg.): Deutschland in der Welt von morgen. Die Chancen unserer Lebens- und Wirtschaftsräume. Hannover, 51-54. = Forschungs- und Sitzungsberichte der ARL 203.

Robertson, C. Y.; Winter, C. (Hrsg.) (2000): Kulturwandel und Globalisierung. Baden-Baden. doi: 10.5445/IR/30012000

Roost, F. (2008): Branding Center. Über den Einfluss globaler Markenkonzerne auf die Innenstädte. Wiesbaden. doi: 10.1007/978-3-531-90992-9

Sassen, S. (1991): The global city. New York, London, Tokyo. Princeton.

Scherer, C. (2006): Korpuslinguistik. Heidelberg. = Kurze Einführungen in die germanistische Linguistik 2.

Schmitt, P. (2007): Raumpolitische Diskurse um Metropolregionen. Eine Spurensuche im Verdichtungsraum Rhein-Ruhr. Dortmund. $=$ Metropolis und Region 1.

Scholz, R. (2010): Die diskursive Legitimation der Europäischen Union. Eine lexikometrische Analyse zur Verwendung des sprachlichen Zeichens "Europa/Europe“ in deutschen, französischen und britischen Wahlprogrammen zu den Europawahlen zwischen 1979 und 2004. Dissertation an der Otto-von-Guericke-Universität Magdeburg.

Simmel, G. (1903): Die Großstädte und das Geistesleben. In: Bücher, K.; Ratzel, F.; von Mayr, G.; Waentig, H.; Simmel, G.; Petermann, T.; Schäfer, G. (Hrsg.): Die Großstadt. Vorträge und Aufsätze zur Städteausstellung. Dresden, 185-206.

Steets, S. (2008): „Wir sind die Stadt!“ Kulturelle Netzwerke und die Konstitution städtischer Räume in Leipzig. Frankfurt am Main.

Stöber, B. (2007): Von „brandneuen“ Städten und Regionen Place Branding und die Rolle der visuellen Medien. In: Social Geography 2, 47-61.

Swyngedouw, E. (2004): Globalisation or 'glocalisation'? Networks, territories and rescaling. In: Cambridge Review of International Affairs 17, 1, 25-48. doi: 10.1080/0955757042000203632

Taylor, P. J.; Ni, P.; Derudder, B.; Hoyler, M.; Huang, J.; Pain, K.; Witlox, F.; Yang, X.; Bassens, D.; Shen, W. (2010): Command and Control Centres in the World Economy. In: Taylor, P. J.; Ni, P.; Derudder, B.; Hoyler, M.; Huang, J.; Witlox, F. (Hrsg.): Global Urban Analysis. A Survey of Cities in Globalization. London, 17-21.
Taylor, P. J.; Walker, D. R. F.; Beaverstock, J.V. (2002): Firms and their Global Service Networks. In: Sassen, S. (Hrsg.): Global networks, linked cities. New York, 93-115.

van den Berg, L.; Drewett, R.; Klaassen, L. H.; Rossi, A.; Vijverberg, C. H. T. (1982): Urban Europe. A study of growth and decline. Oxford. doi: 10.1016/C2013-0-03056-3

Volgmann, K. (2014): Entwicklung metropolitaner Funktionen im polyzentralen deutschen Städtesystem - Raummuster der Konzentration und funktionalen Spezialisierung. In: Raumforschung und Raumordnung 72, 1, 21-37. doi: 10.1007/ s13147-013-0264-5

Wiechmann, T. (2009): Raumpolitische Diskurse um Metropolregionen in Europa - Eine Spurensuche. In: Knieling, J. (Hrsg.): Metropolregionen. Innovation, Wettbewerb, Handlungsfähigkeit. Hannover, 101-132. = Forschungs- und Sitzungsberichte der ARL 231.

Wirth, L. (1938): Urbanism as a Way of Life. In: American Journal of Sociology 44, 1, 1-24. doi: 10.1086/217913

Zohlen, G. (1995): Metropole als Metapher. In: Fuchs, G.; Moltmann, B.; Prigge, W. (Hrsg.): Mythos Metropole. Frankfurt am Main, 23-34.

Zukin, S. (1996): Space and Symbols in the Age of Decline. In: King, A. (Hrsg.): Re-Presenting the City. Ethnicity, Capital and Culture. Basingstoke, 43-59.

Zukin, S. (2010): Stadtkultur - Auf der Suche nach Authentizität. In: Hannemann, C.; Glasauer, H.; Pohlan, J.; Pott, A.; Kirchberg, V. (Hrsg.): Stadtkultur und Kreativität. Jahrbuch StadtRegion 2009/10. Opladen, 45-63. 